ص ص[จq-

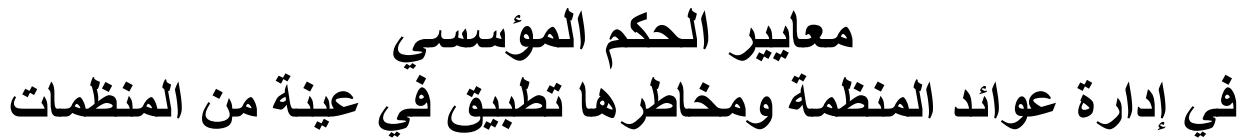

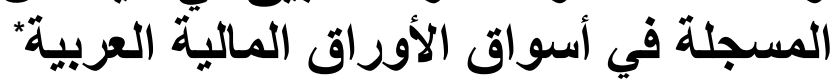

\author{
دعاء نعمان الحسيني \\ مدرس -قسم العلوم المالية والمصرفية \\ كلية الإدارة والاقتصاد -جامعة الموصل \\ duaahusaeny@yahoo.com
}

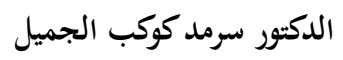 \\ أستاذ -قسم العلوم المالية والمصرفية الحميل \\ كلية الإدارة والاقتصاد -جامعة الموصل
}

Sarmad_aljamil53@yahoo.com

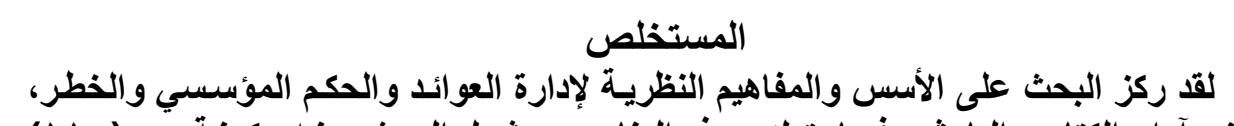

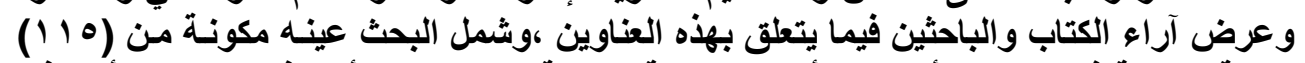

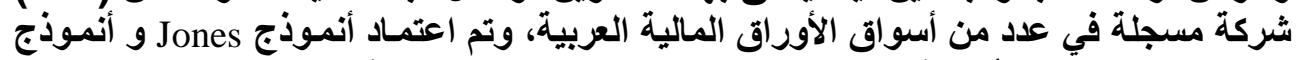
Modified Jones

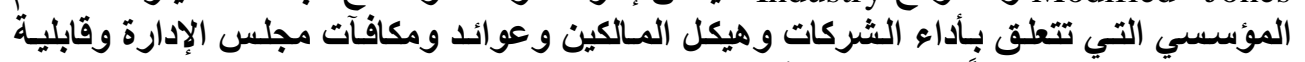

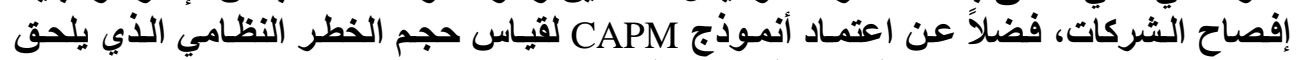

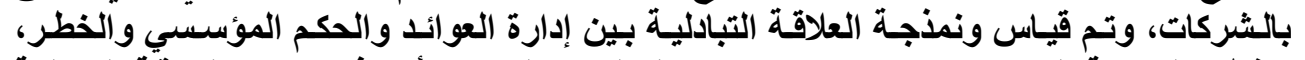

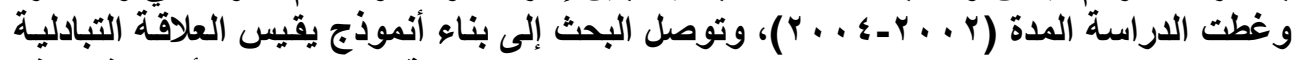

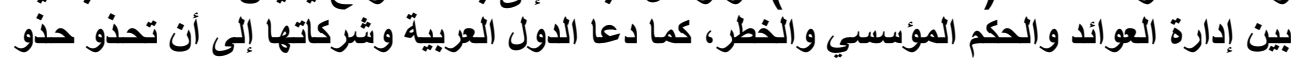

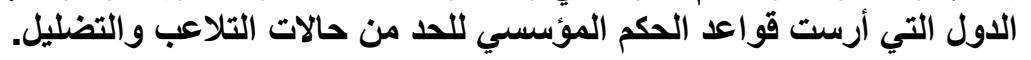
الكلمات المفتاحية: إدارة العوائد، الحكم المؤسسي، الخطر.

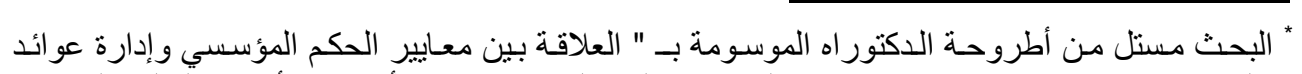

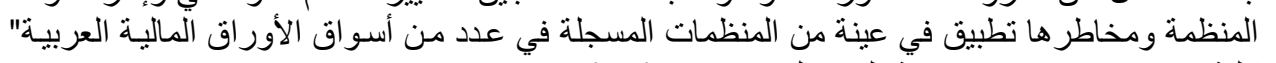

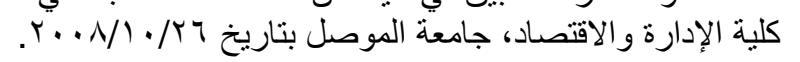

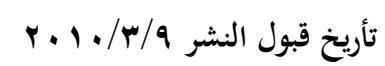

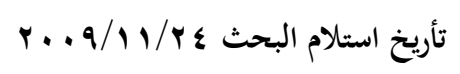




\title{
The Governance Criteria in Earnings Management of Organization and its Risks: An Application on Sample of Registered Organizations in Arabic Stock Markets
}

\author{
Sarmad K. Al-Jameel (PhD) \\ Professor \\ Department of Financial and Banking \\ Sciences \\ University of Mosul
}

\author{
Duaa N. Al-Hussaini \\ Assistant Lecturer \\ Department of Financial and Banking \\ Sciences \\ University of Mosul
}

\begin{abstract}
The research concentrated on the theoretical concepts of earning management, governance and risk. The research showed insights of researchers and scholars related to these titles. The research contains a sample consisted of (115) companies recorded in a number of Arab stock markets and (Jones model, Modified Jones and Industry model) were applied to measure the earning management. As well as the research modeled the variables of governance which related to companies performance, ownership structure, returns, rewards of management board and the ability of companies announcement. In addition to the adoption of CAPM model to measure systematic risk which affect the companies. The retrospective modeled between earnings management, governance and risk were modeled. The research covered the period between (2002-2004) and reached to construct a model of measuring the exchange relation between earnings management, governance and risk, and invite the Arab countries and their companies to follow suit the countries which established the governance rules, to declare play and maneuver cases.
\end{abstract}

Key words: Earning Management, Corporate Governance, Risk.

$$
\begin{aligned}
& \text { المقدمة } \\
& \text { يعد موضهوع الحكم المؤسسي Governance توجهاً حديثاً بعكس أسسس ومقومـات }
\end{aligned}
$$

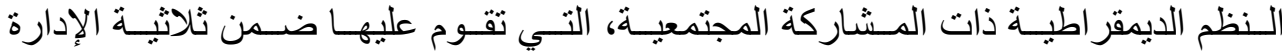

$$
\begin{aligned}
& \text { و المؤسسة و البيئة، و على هذا الأسـاس بات من أهم دوأعي الاهنمـام بقو اعد ومعابير الحكم }
\end{aligned}
$$

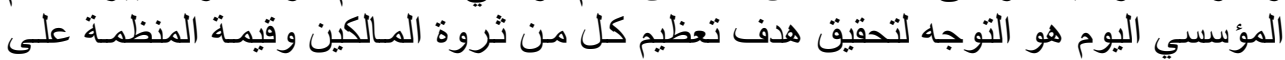

$$
\begin{aligned}
& \text { حدٍ سو اء من خلال تبني الثركة إستر اتيجيات وسياسـات عديدة تستند على إدارة عو ائدها } \\
& \text { Earning Management } \\
& \text { الأطر اف المتعاملة مع الثركة. } \\
& \text { مشكنة البحث } \\
& \text { تبرز مشكلة البحث بشكل واضح عبر تساؤلات عديدة أهمها: }
\end{aligned}
$$

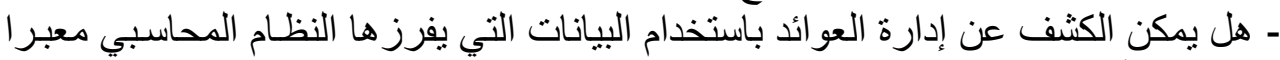

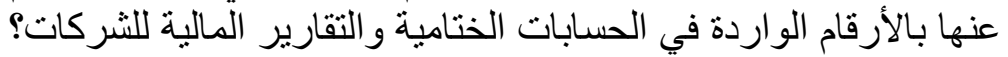

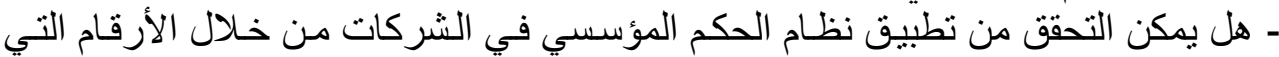

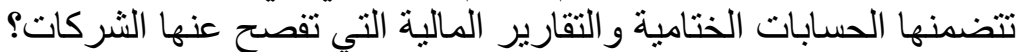

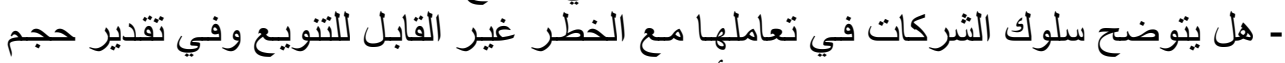

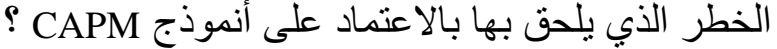

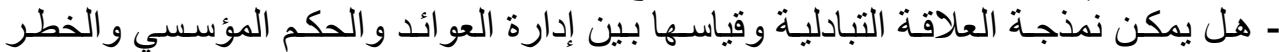

$$
\begin{aligned}
& \text { بالاعتماد على البيانات و المعلومات المحاسبية }
\end{aligned}
$$




\section{الجميل والحسيني] [יר]}

\section{أهمية البحث}

إن التوجه في إدارة عو ائد الثركة وتأطير أخطار ها على وفق قو اعد ومعايير الحكم

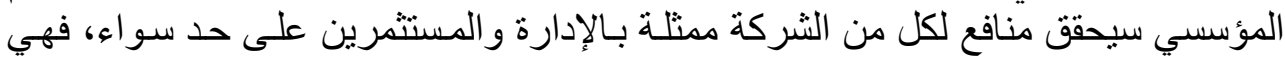

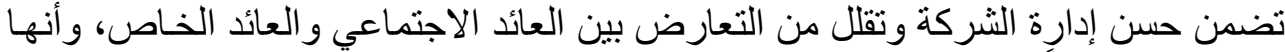

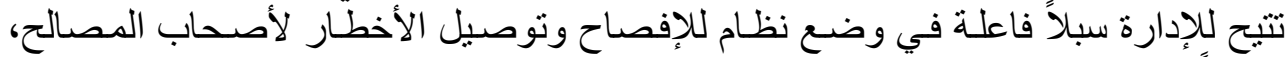

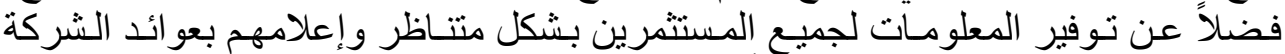

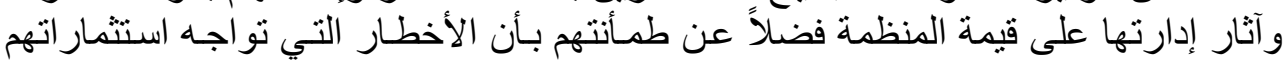

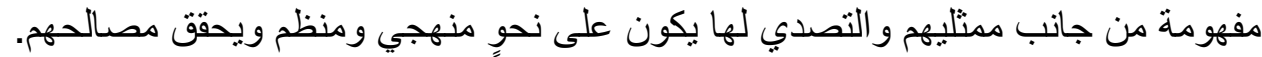

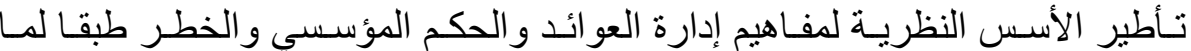

$$
\text { أوردته الأدبيات الإدارية والإستر اتيجية. }
$$

"يمكن نمذجة العلاقة بين الحكم المؤسسي و إدارة العو ائد و المخـاطر التي تواجهها

$$
\text { فرضية البحث }
$$

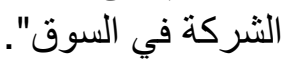

$$
\text { الأسس النظرية للبحث }
$$

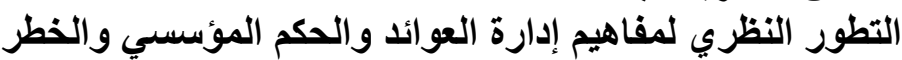

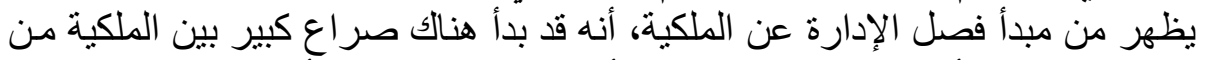

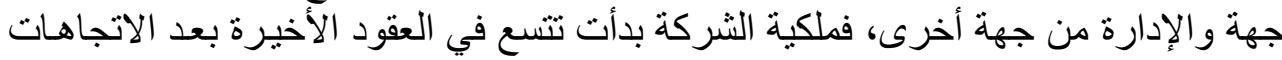

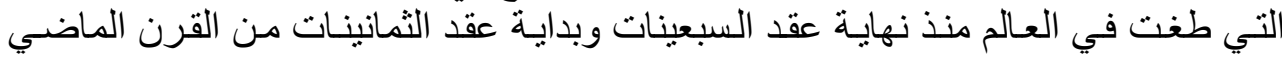

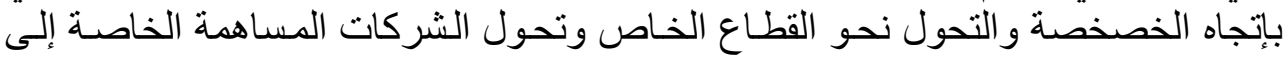

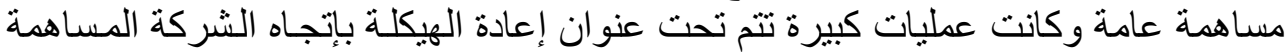

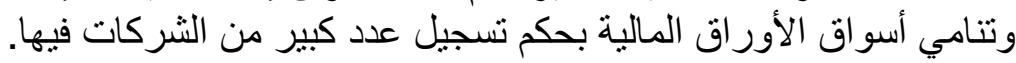

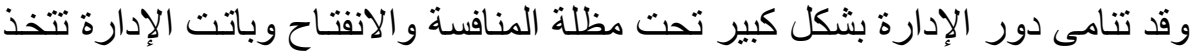

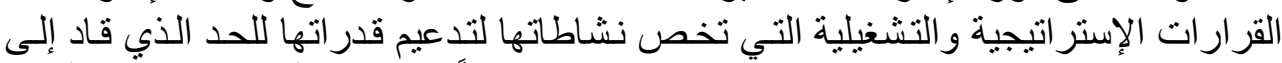

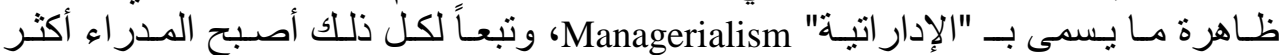

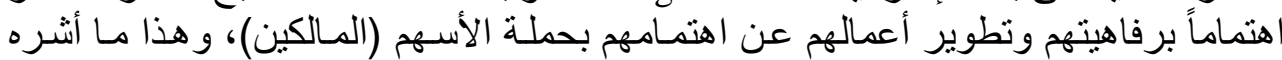
Pike and Neale

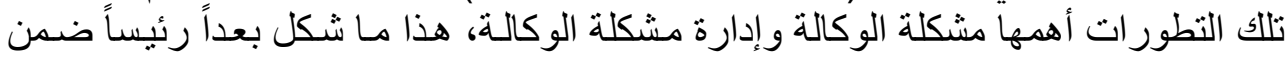

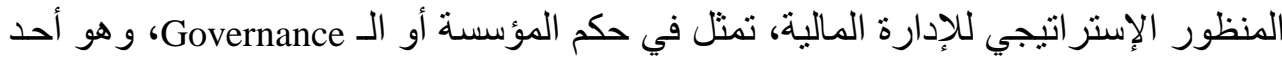

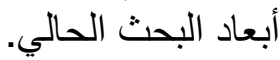

البعد الأوّل- نشوء وتطور إدارة العوائد

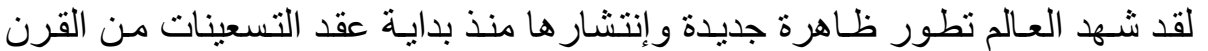

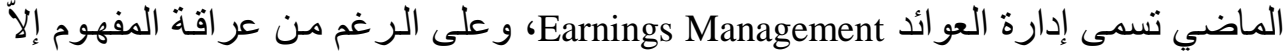




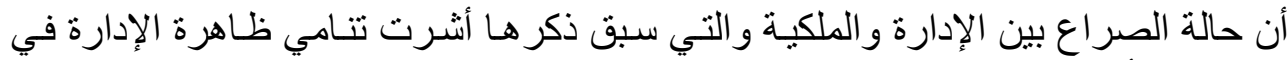

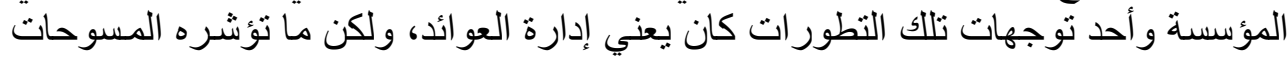

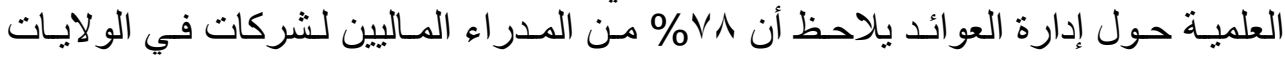

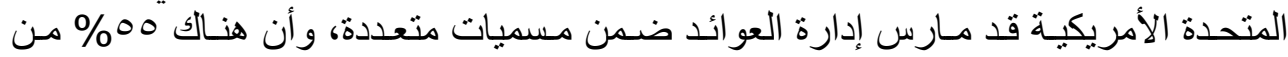

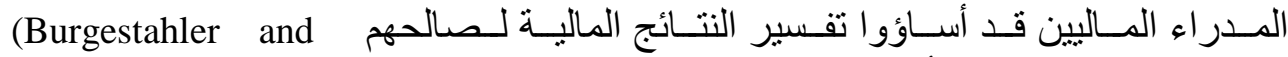

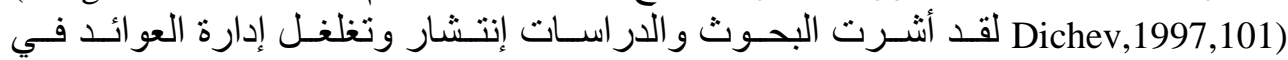

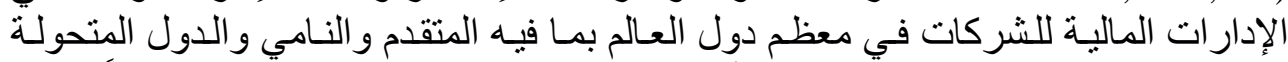

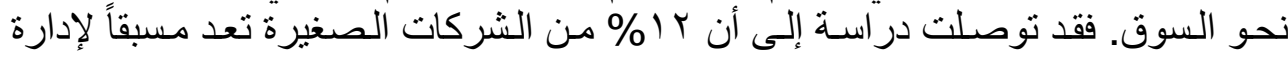

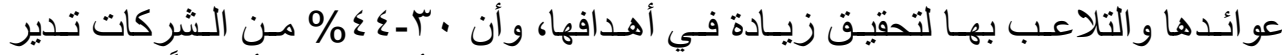

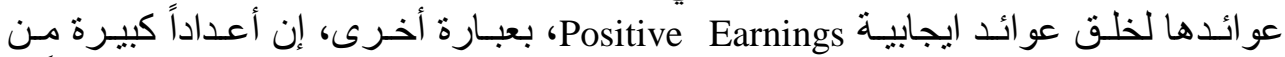

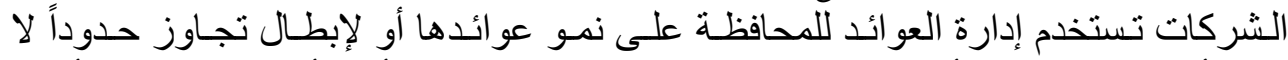

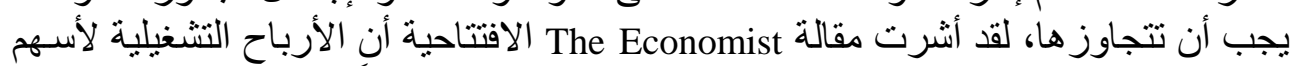

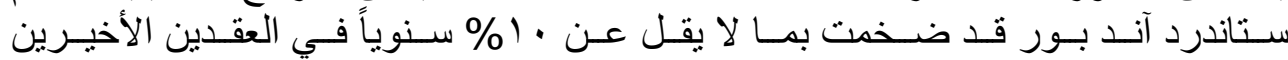
. (Economist, 2001, 72) ومن هنا بدأت الهيئات المهنية الخاصة بالمعايير المحاسبية تصدر المزيد من المعايير

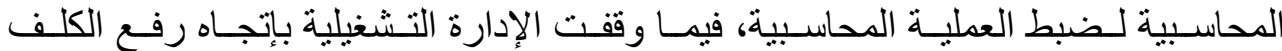

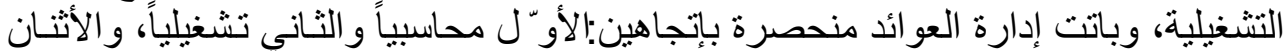

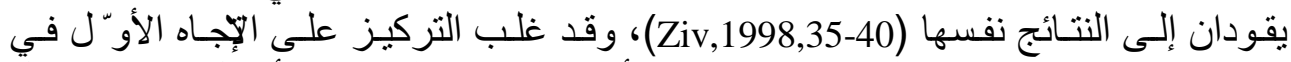

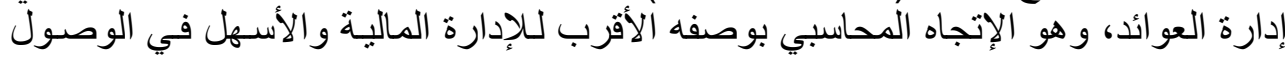
للنتائج.

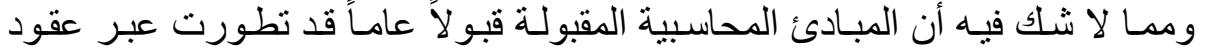

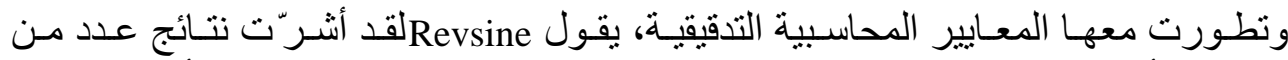

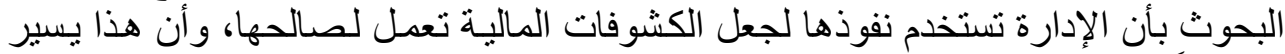

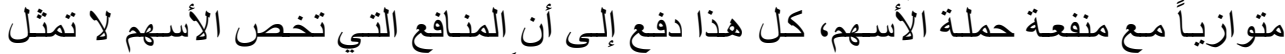

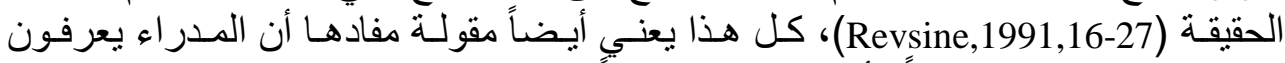

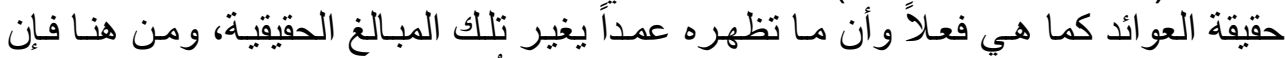

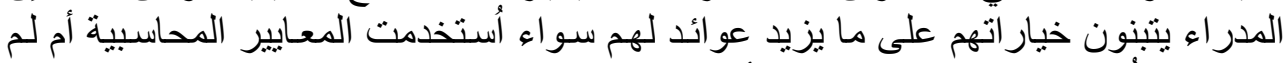

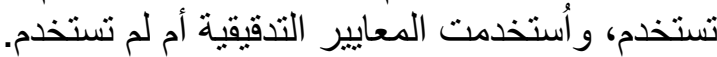

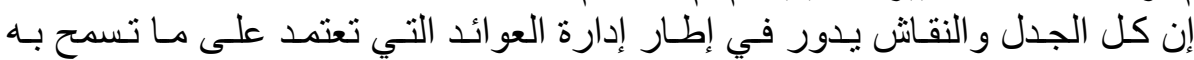

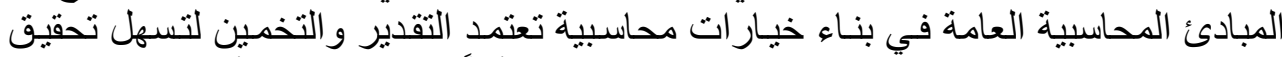

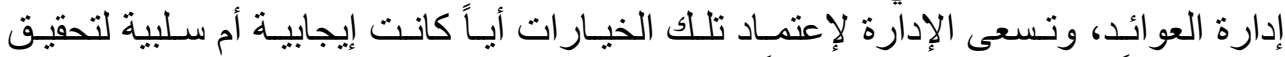
منافعها أو لاً و ونفعة حملة الأسهر ثانياً.

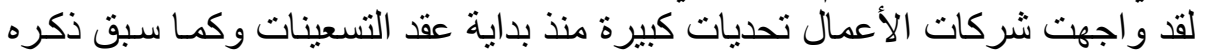

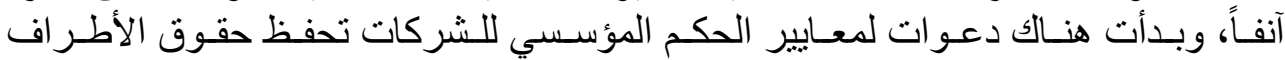

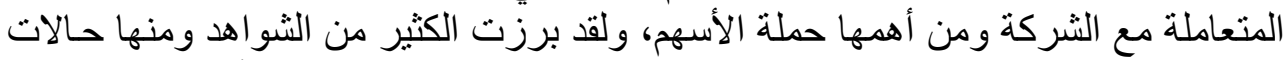

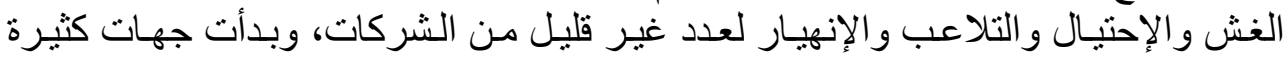

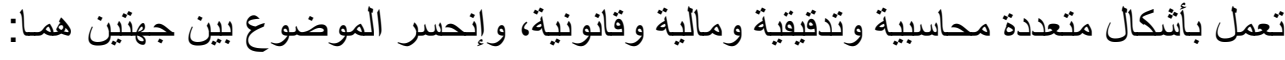




\section{الجميل والحسيني[r]}

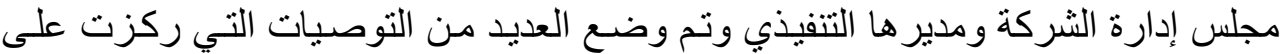
الفصل التام بين مجلس الإدارة والمدير التنفيذي، وبدأ التركيز على الإسئ الإستر اتيجية والأداء

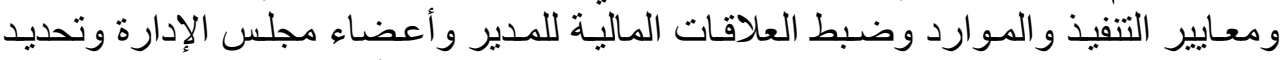

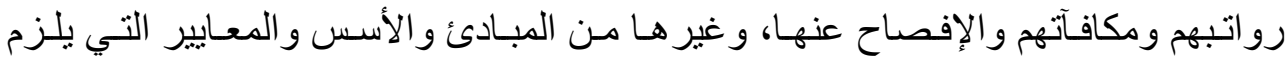

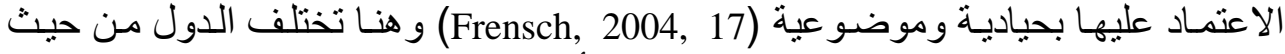

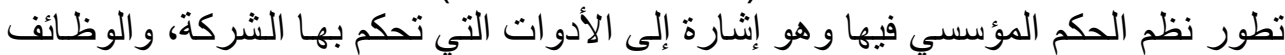

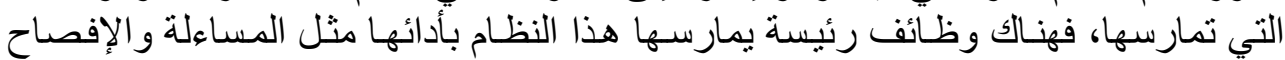

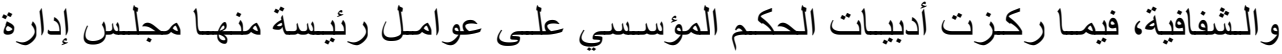

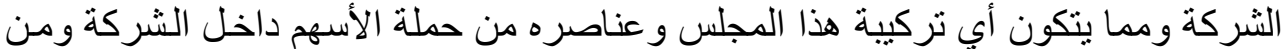

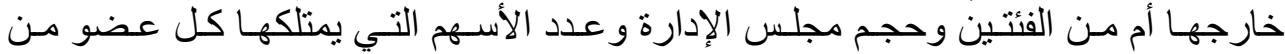

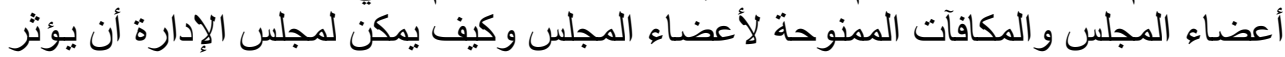

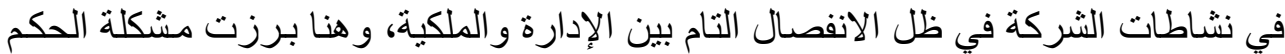

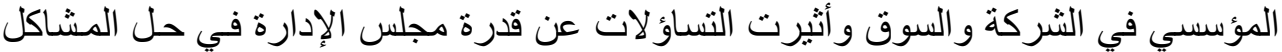

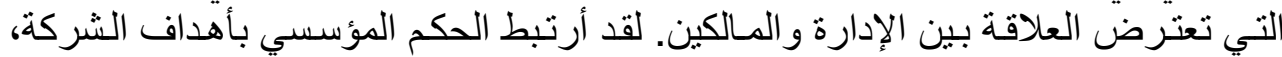

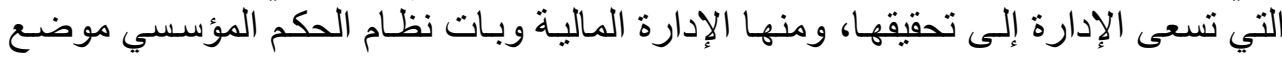

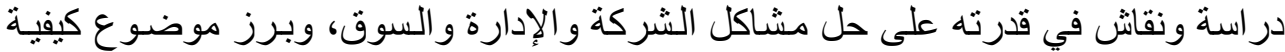

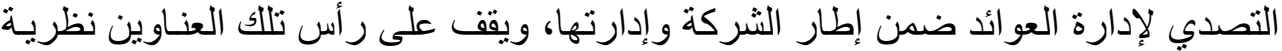

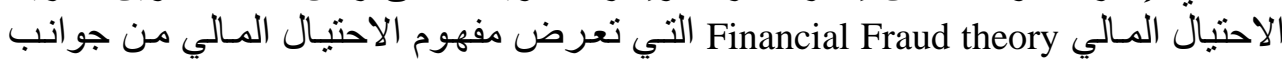

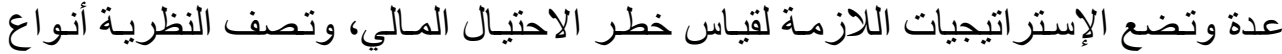

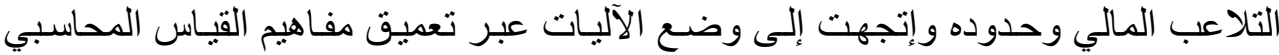
و التدقيقي و القانوني.

البعد الثالث- نشوء وتطور مفهوم الخطر

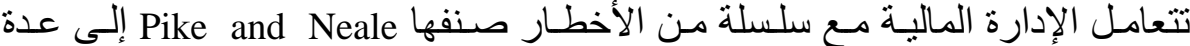

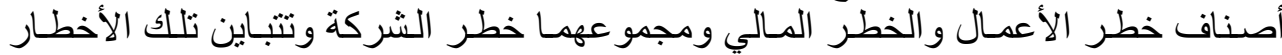

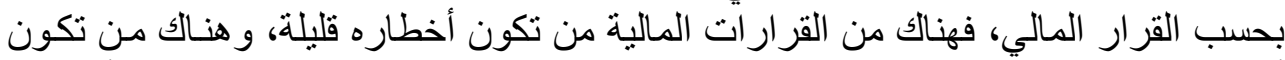

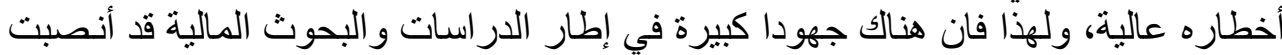

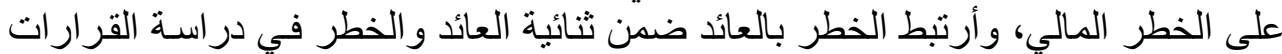

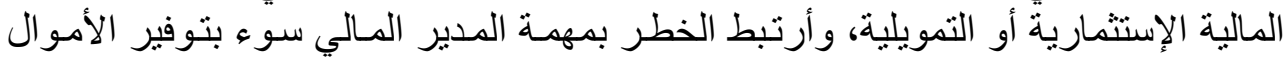

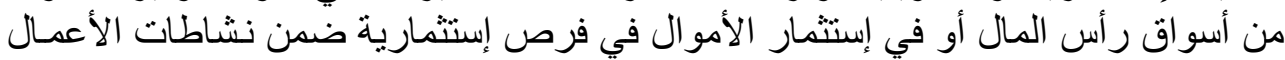

.(Pike and Neale, 1993, 17-18)

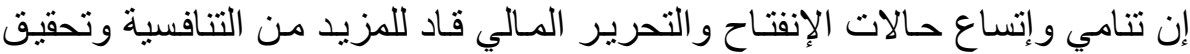

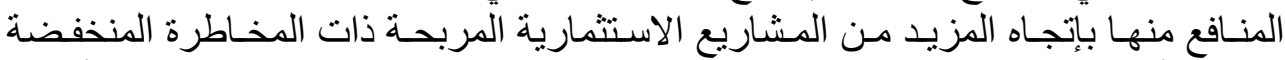

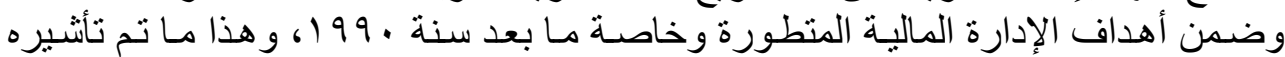

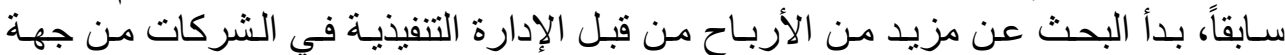

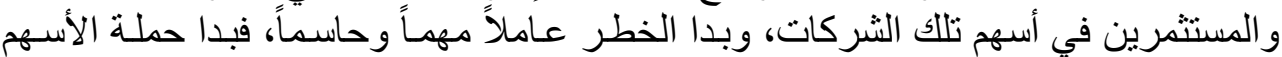

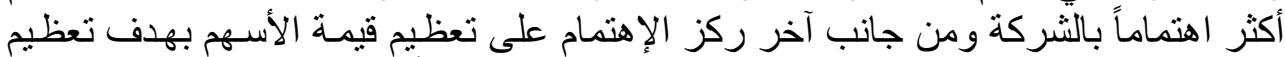

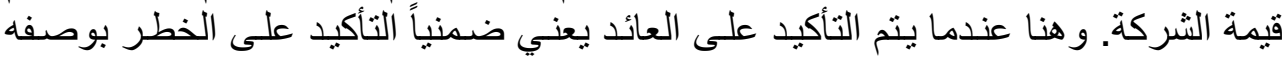
الخسارة المنوقعة ومحاولة تجنبها يعني تعظيم العائد (Pike and Neale, 1993, 23). 


\section{الحكم المؤسسي- خط الدفاع الأوّل}

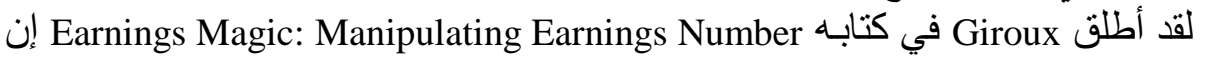

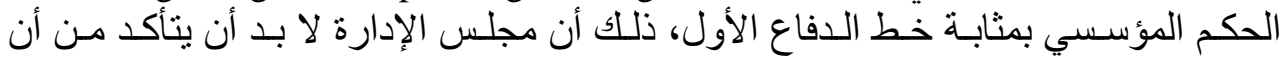

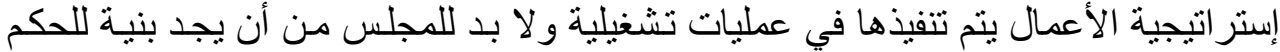

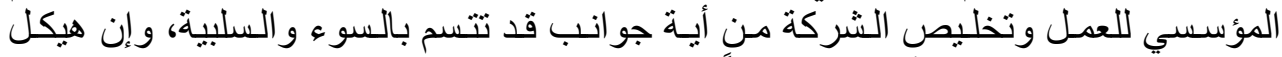

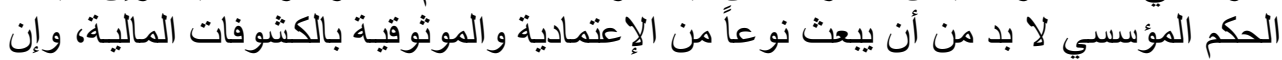

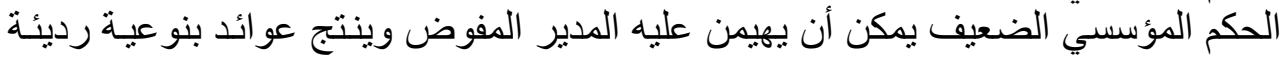

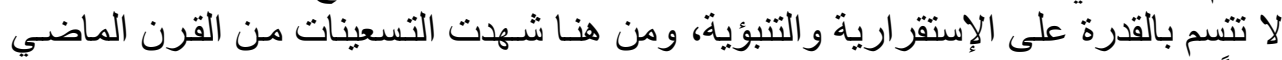

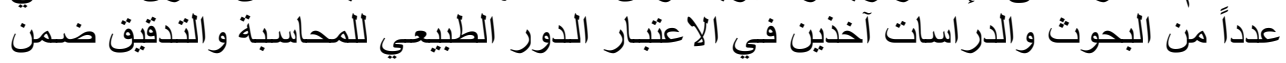

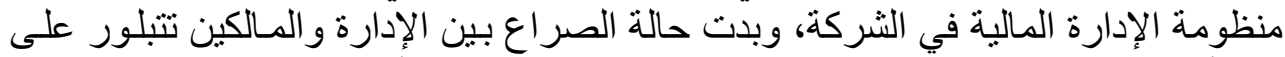

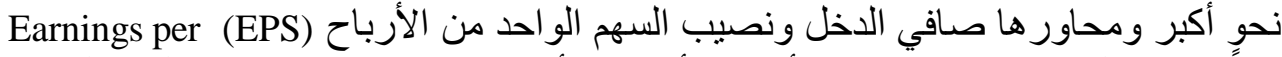
share

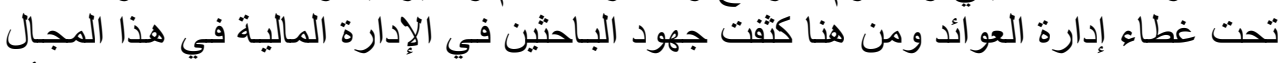

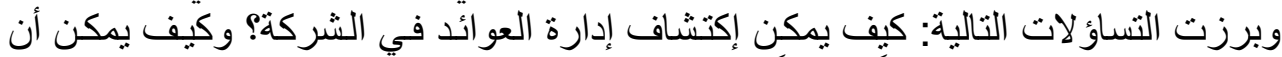

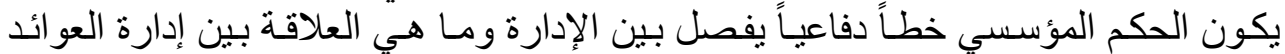

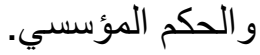

إدارة العو ائد ونماذج قياسها

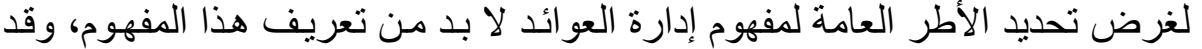

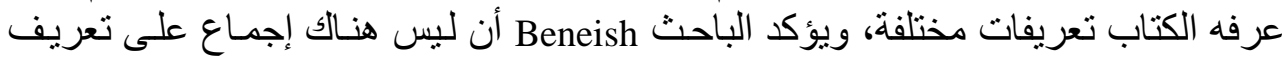

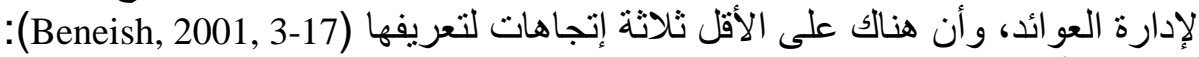

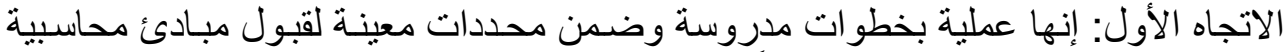

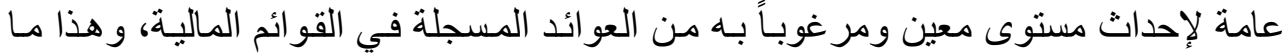

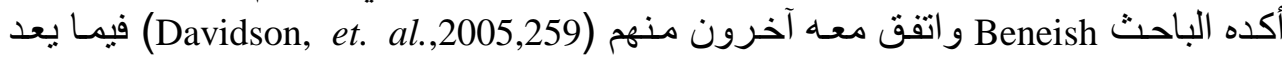

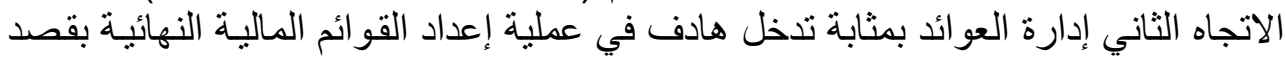

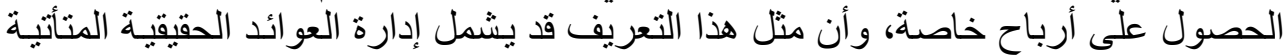

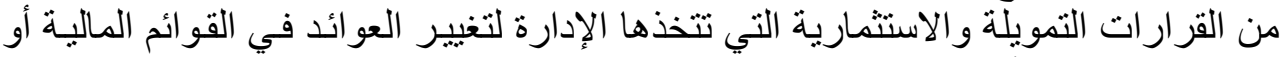

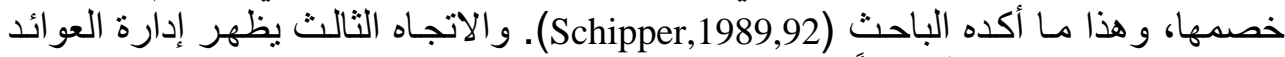

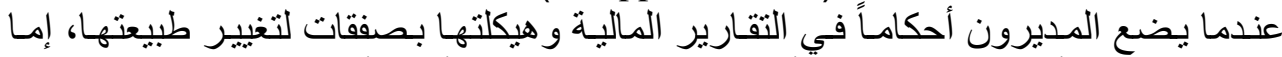

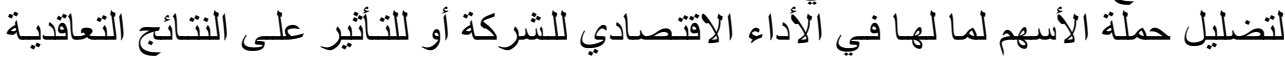

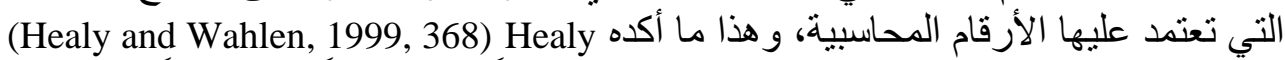

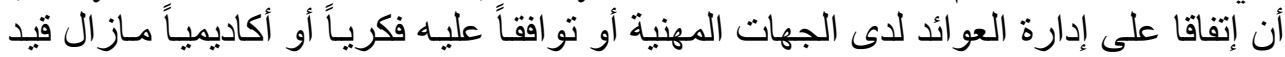

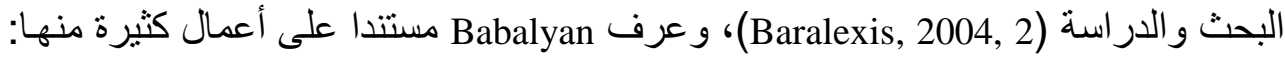
Kinney and و و (Stolowy and Breton,2000)و (Mulford and Comiskey,2002)

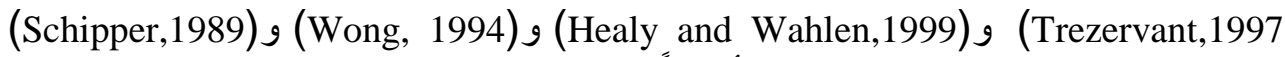

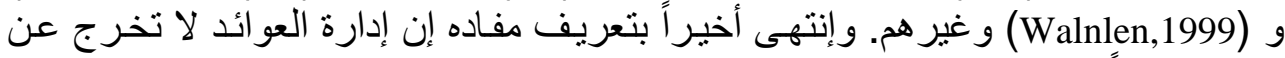
كونها تلاعباً في حساب المستحقات وخيار ات الإدارة في استخدام السياسات و الطر ائق في 
الجميل والحسيني[0]

ومليات التقدير و التخمين، ومن تلك الأنشطة مـا يندرج ضمن الحيل و الخدع بقصد معين وأغر اض بعيدة الأمد (Babalyan, 2004, 6-16).

نماذج إدارة العوائد

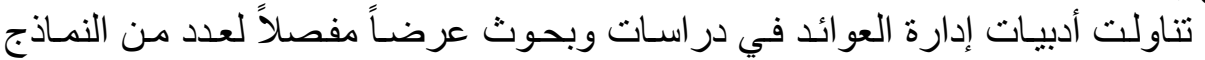

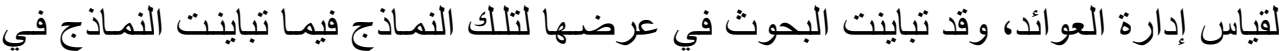

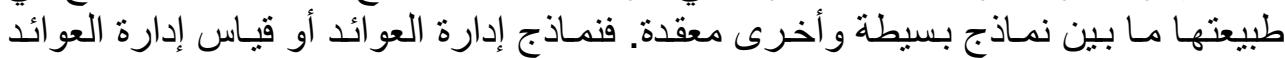

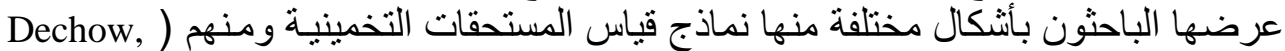
(et al, 1995, 8 قياس المستحقات التخمينية ضمن مجموع المستحقات كمدخل لقياس إدارة العو ائد.

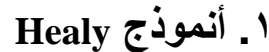

لقد وضع Healy أنموذجها في بحثنه الذي قدمـه عـام 910 القياس إدارة العوائد من

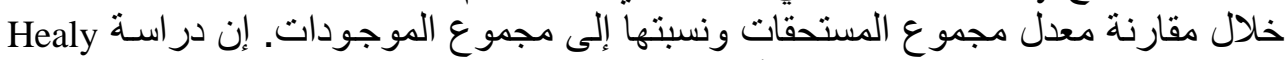
إعتمدت التنبؤ بالعائدات النظامية في أية مدة زمنية معينة.

$$
\mathrm{NDA}=\frac{\sum \mathrm{TA}_{\mathrm{t}}}{\mathrm{T}_{\mathrm{t}-*}}
$$

NDA = المستحقات غير التخمينية

t الزمن

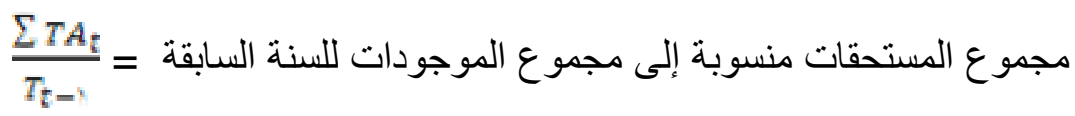

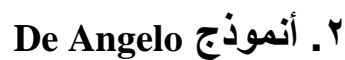

قدم De Angelo انموذجه في عام 9 أن 1، حيث إعتمد على إفتر اض أن المستحقات

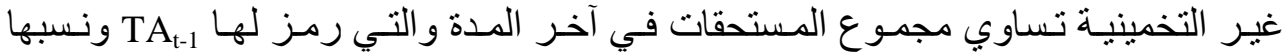

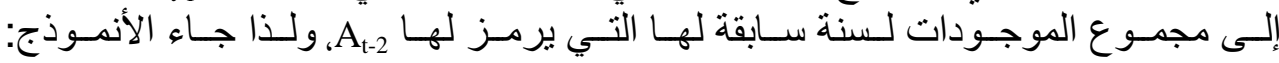

$$
\begin{aligned}
& \mathrm{NDA}=\frac{\mathrm{TA}_{\mathrm{t}-\mathrm{*}}}{\mathrm{A}_{\mathrm{t}-\mathrm{T}}} \\
& \text { وقد وضع De Angelo أنموذجه بعد در اسة Healy وتقديره للمدة الزمنية. }
\end{aligned}
$$$$
\text { (De Angelo, 1986, 400-420) }
$$

\section{Jones Model أنموذج جونز}

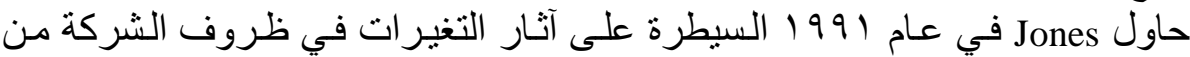
خلال المستحقات غير التخمينية وعرض أنموذجه كما يأني: $\mathrm{NDA}_{t}=\mathrm{a}_{1}\left(1 / \mathrm{A}_{\mathrm{t}-1}\right)+\mathrm{a}_{2}\left(\Delta \operatorname{Rev}_{\mathrm{t}} / \mathrm{A}_{\mathrm{t}-1}\right)+\mathrm{a}_{3}\left(\mathrm{PPE}_{\mathrm{t}} / \mathrm{A}_{\mathrm{t}-1}\right)$ 
NDA = المستحقات غير التخمينية

A Aموع الموجودات للسنة السابقة

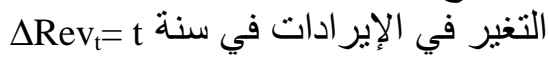

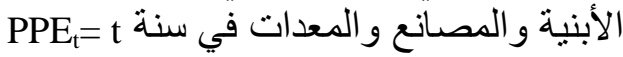

و أكد انه للحصول على المعاملات

$\mathrm{TA} / \mathrm{A}_{\mathrm{t}-1}=\mathrm{a}_{1}\left(1 / \mathrm{A}_{\mathrm{t}-1}\right)+\mathrm{a}_{2}\left(\Delta \operatorname{Rev}_{\mathrm{t}} / \mathrm{A}_{\mathrm{t}-1}\right)+\mathrm{a}_{3}\left(\mathrm{PPE}_{\mathrm{t}} / \mathrm{A}_{\mathrm{t}-1}\right)+\mathrm{e}_{1}$

حيث المعاملات

$$
\text { تمنثل البواقي. }
$$

\section{؛ ـ أنموذج جونز المعدل Modified Jones Model}

إعتمد الأنموذج على سابقه أنموذج Jones وباستخدام المعادلة الآتية:

$\mathrm{NDA}_{\mathrm{t}}=\mathrm{a}_{1}\left(1 / \mathrm{A}_{\mathrm{t}-1}\right)+\mathrm{a}_{2}\left[\left(\Delta \operatorname{Rev}_{\mathrm{t}}-\Delta \operatorname{Rec}_{\mathrm{t}} / \mathrm{A}_{\mathrm{t}-1}\right)\right]+\mathrm{a}_{3}\left(\mathrm{PPE}_{\mathrm{t}} / \mathrm{A}_{\mathrm{t}-1}\right)$

$\mathrm{NDA}_{\mathrm{t}}=\mathrm{t}$

1/A نسبة ا إلى إجمالي الموجودات لسنة سابقة

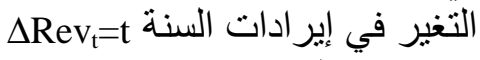

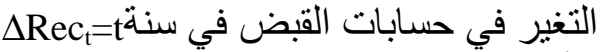

الأبنية والمصانع و المعدات في سنة في فئة

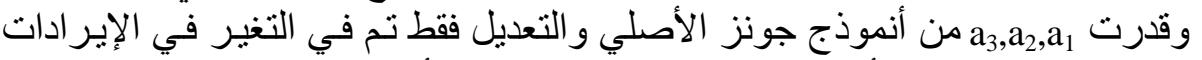

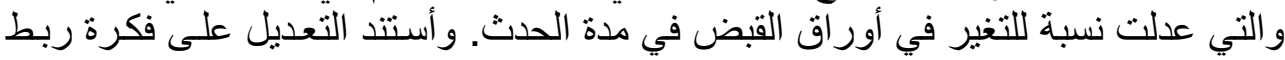

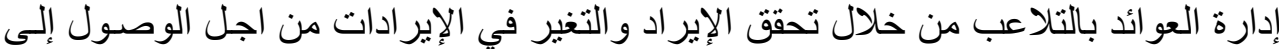

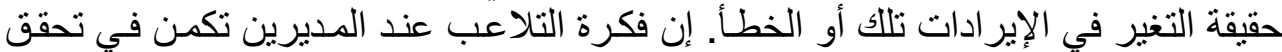

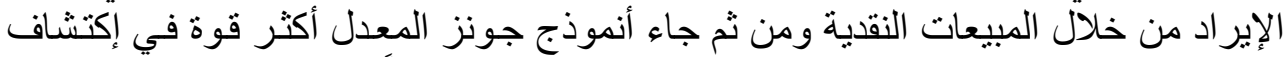

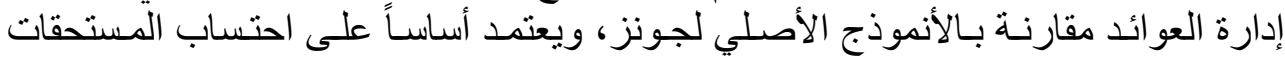

هـ الأنموذج الصناعي The Industrial Model

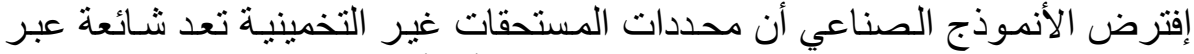

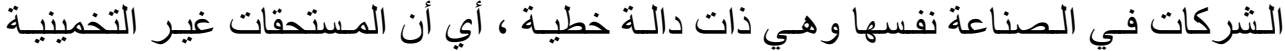

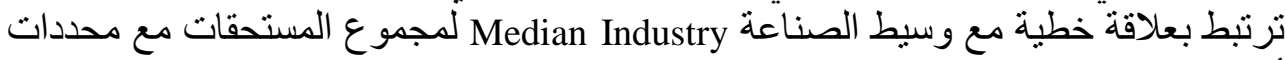

$\mathrm{NDA}_{\mathrm{t}}=\mathrm{B}_{1}+\mathrm{B}_{2}$ median $\left(\mathrm{Ta}_{\mathrm{t}} / \mathrm{A}_{\mathrm{t}-1}\right)$

المستحقات غير التخمينية= المينة

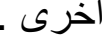

إجمالي المستحقات في سنة إنة

إجمالي الّي الموجودات لسنة سابقة

Bملمات خاصة بالأنموذج

يعتمد قياس NDA (المستحقات غير التخمينية) على مـا ورد في أنموذج جونز والتودي

نسبت إلى مجموع المستحقات (Zhang,2002,13). 
7. النماذج المقطعية Cross Sectional Models

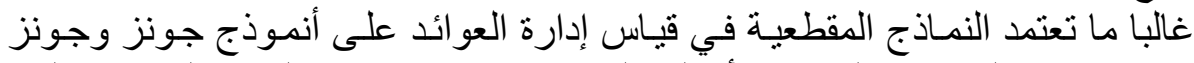

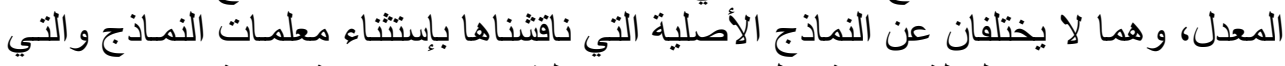

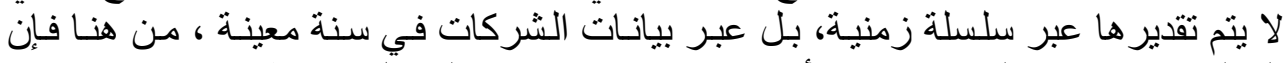

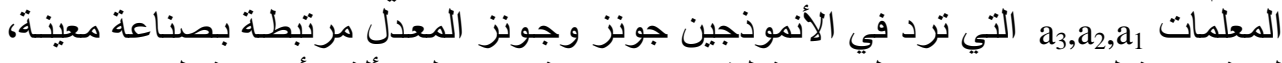

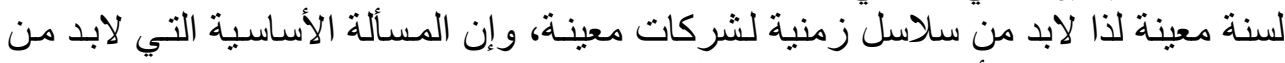

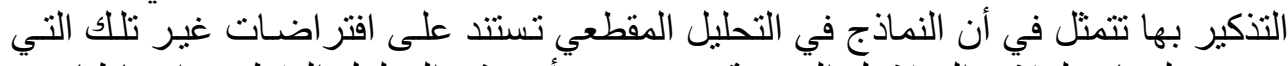

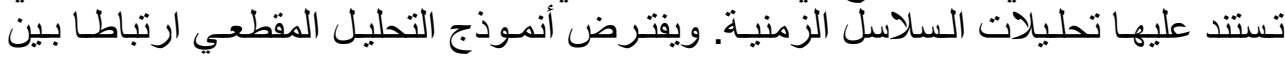

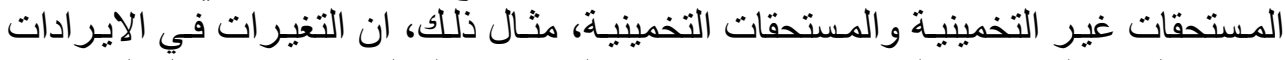

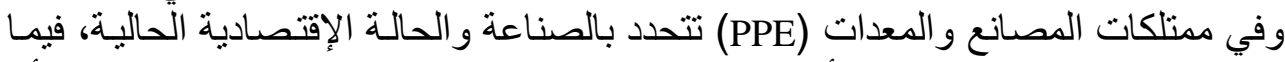

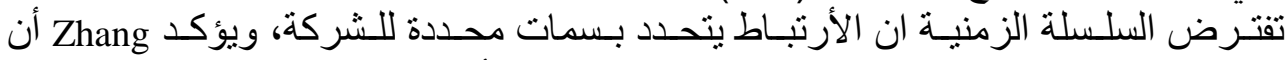
المستحقات التخمينية تحتسب بإستخدام التحليل المقطعي لأنموذج جونز وجونز المبند المعدل.

\section{The Forward-Looking Modified Jones Model}

\section{V. أنموذج جونز المعدل ذو المنظور الامامي}

ويستند الباحث في عرضـه لهذا الأنمـوذج على مـا طرحسه (Dechow,et al,2002) وسماه المنظور الأمامي للمستحقات غير التخمينية وتظهر معادلته كما يأتي:

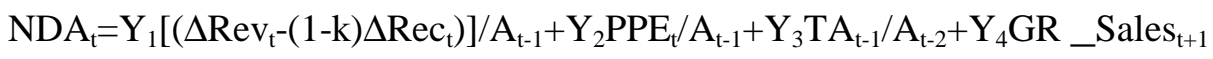

ميث معامل الأرتباط من خلال إنحدار تغير في أوراق القبض مع التغير في الاير ادات

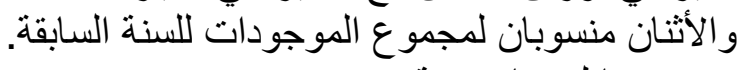

GR_Sales $=$ منسوبا لمبيعات سنة $\mathrm{GR}=$ معدل النمو

$$
\text { يتضمن الأنموذج ثلاثة تعديلات لأنموذج جونز المعدل هي: }
$$

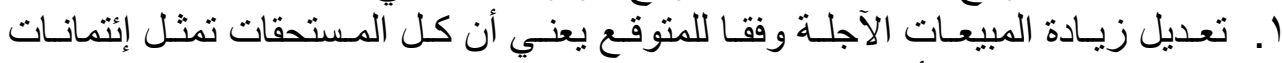

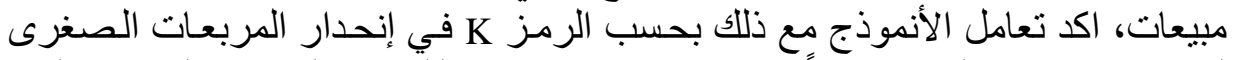

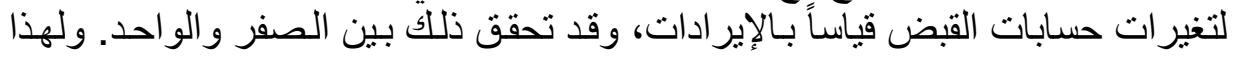

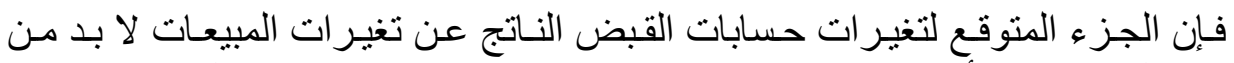

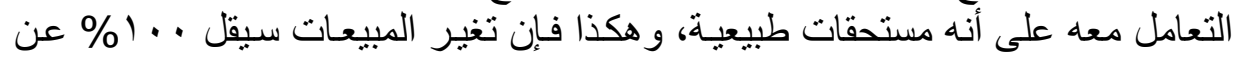

$$
\text { زيادة حسابات القبض النه }
$$

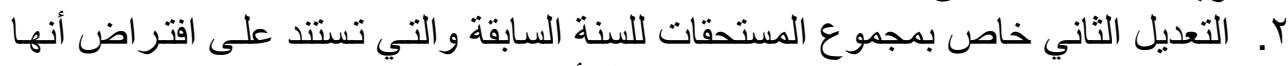

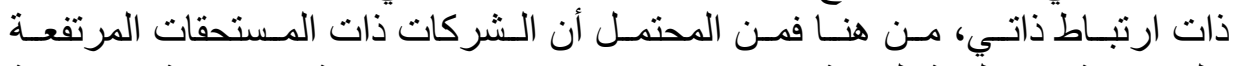

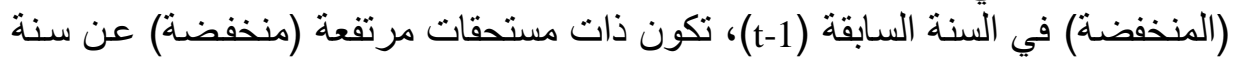

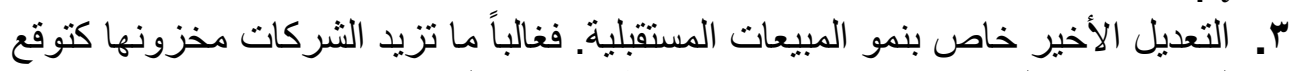

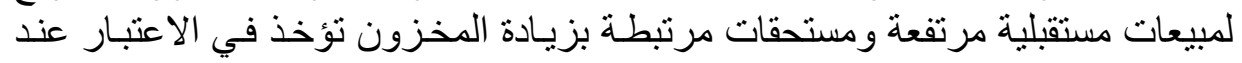


إدارة العو ائد، ولكن رد فعل الإدارة يتمثل في التنبؤ بالمبيعـات ونموهـا في المستقبل

(Zhang, 2002, 14-15)

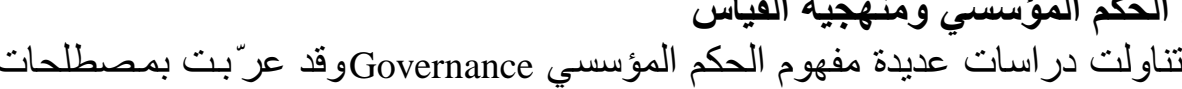

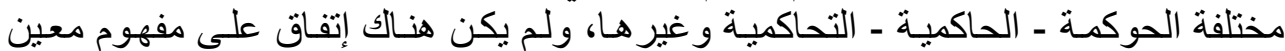

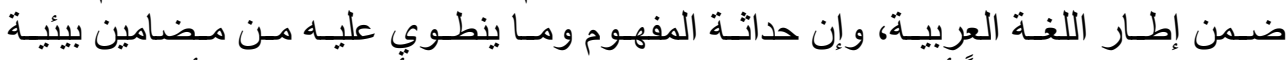

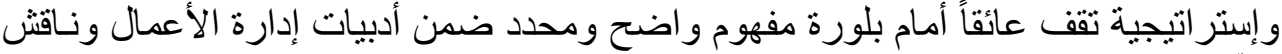

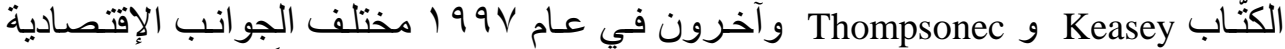

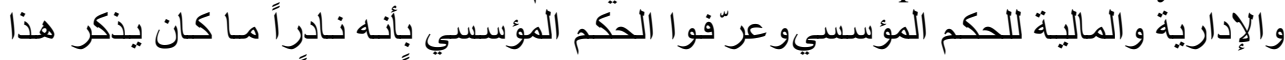

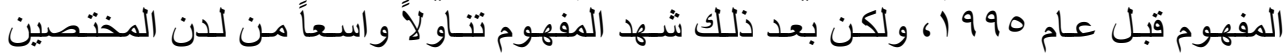

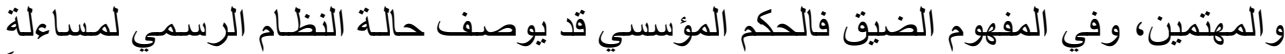

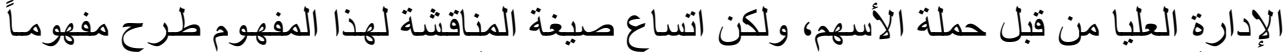

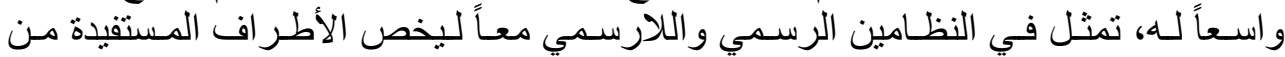
المنظمة في المجتمع، ومن هنا يمكن القول بان الحكم المؤسسي يتمثل في هيكل Structure

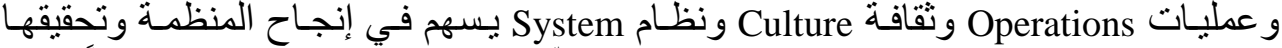

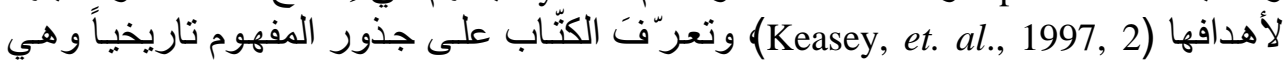

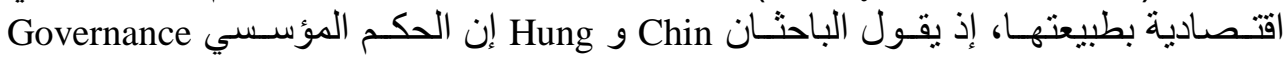

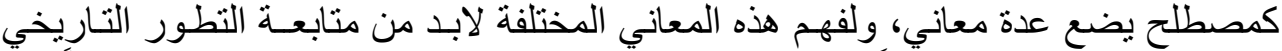

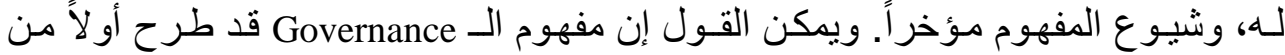
قبل البنك الدولي، وقد اعتمد منذ ذلك الحين من قبل فيل عدد كبير من المؤسسات الدولية، وكل

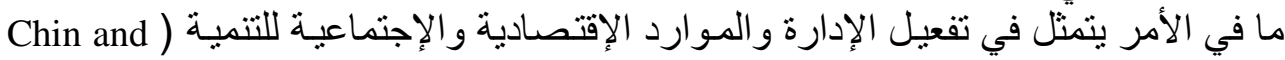

.(Hung, 2004, 2-3

\section{الخطر ودور أنموذج تسعير الموجود الرأسمالي في قياسه}

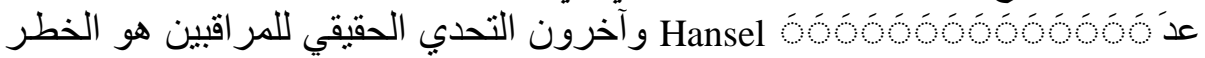

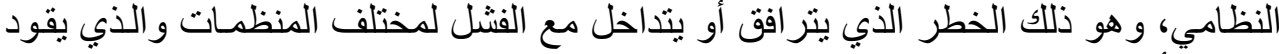

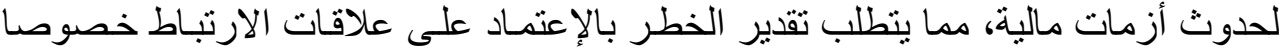

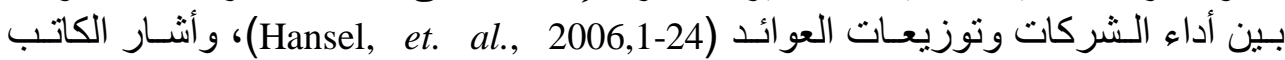
Gitman

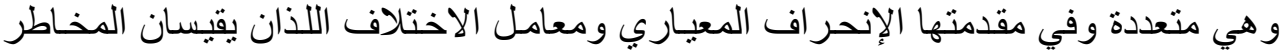

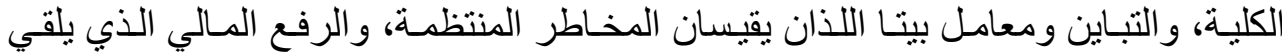

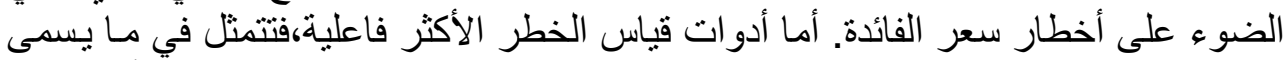

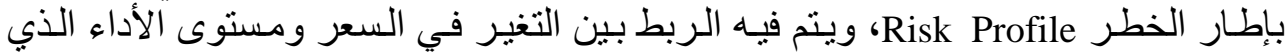

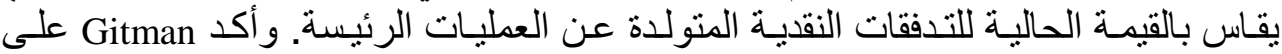

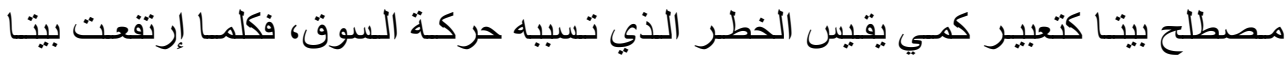

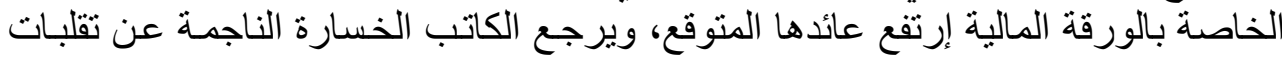

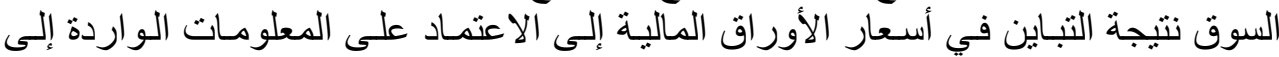

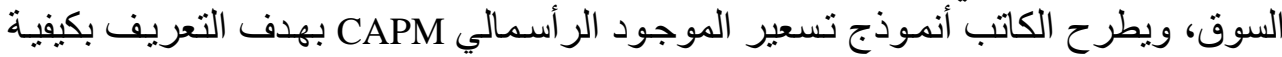




\section{الجميل والحسيني[9]}

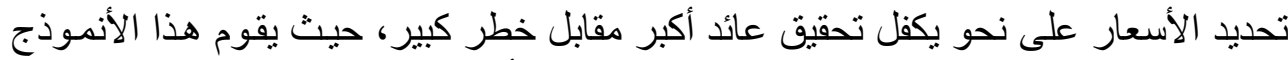

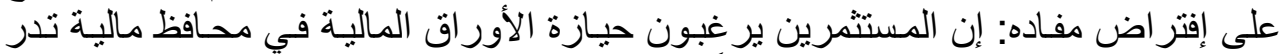

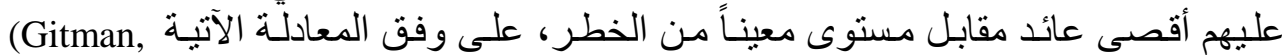

$\mathrm{K}_{\mathrm{i}}=\mathrm{R}_{\mathrm{f}}+\left[b_{\mathrm{i}}^{*}\left(\mathrm{~K}_{\mathrm{m}}-\mathrm{R}_{\mathrm{f}}\right)\right]$

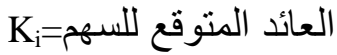

Re

bi= معامل بيتا ويمثل الأخطار المنتظمة للورقة المن المخالية

Kائد السوق = Kن

تأطير العلاقة النظرية بين إدارة العوائد والحكم المؤسسي والخطر

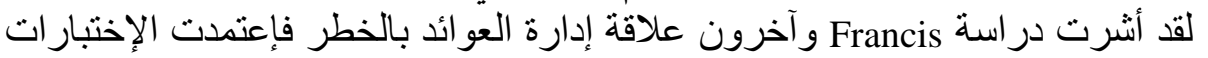

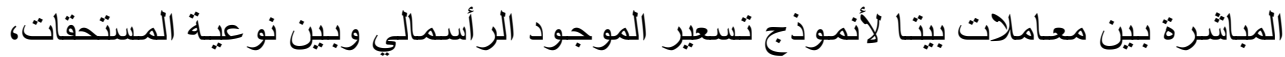

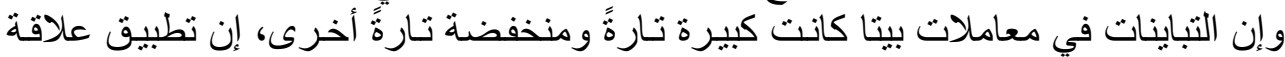

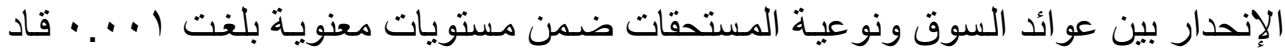

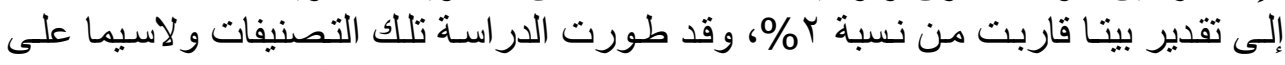

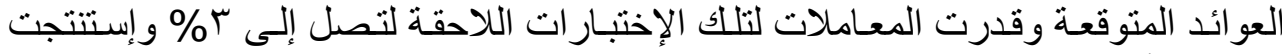

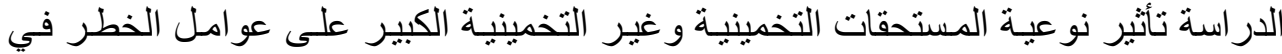

السوق (Francis, et. al., 2004, 2). يقول الباحثان Schaller و Chirinko إن لمشاكل الحكم المؤسسي تأثثر مباشر وسريع

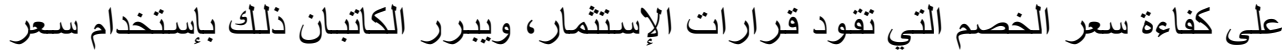

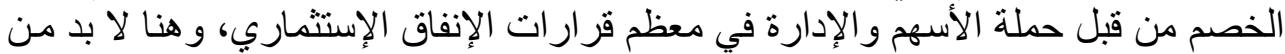

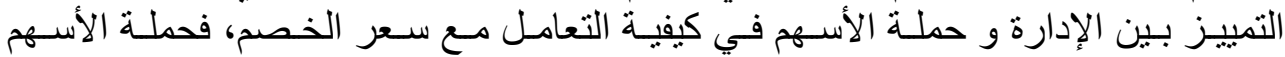

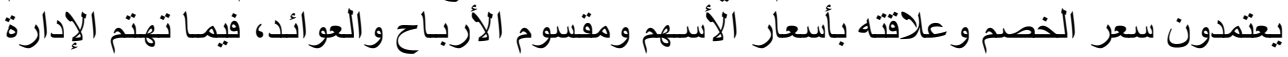
بسعر الخصم الذي يخص الإنفاق الاستثماري (Chirinko and Schaller, 2004, 2).

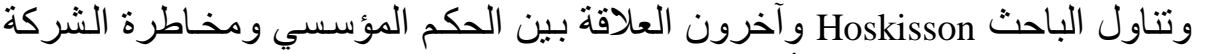
في إطار علافة منهجية في كيفية تأثثر نظام الحكم المؤسسي في الثركةٍ على خطر الشركة (Hoskisson, et. al. 2004, 293-315) منهجية البحث ومعالجة البيانات وتوصيفها

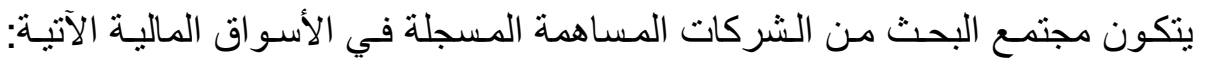

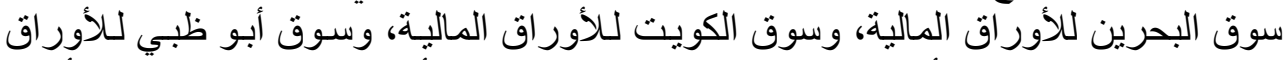

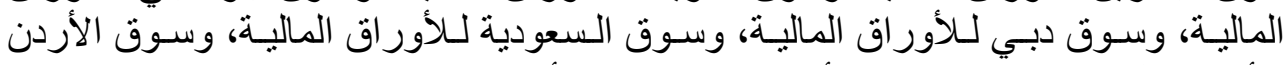

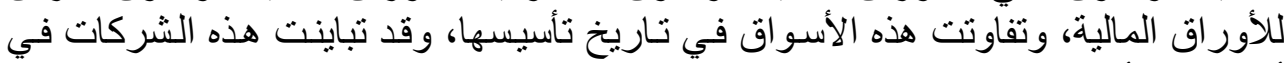

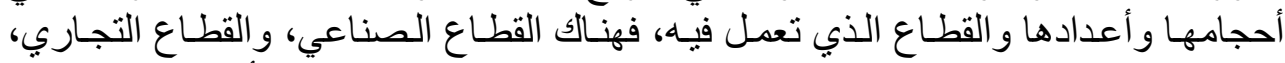

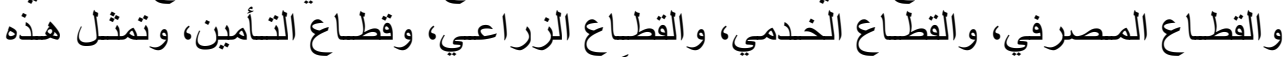

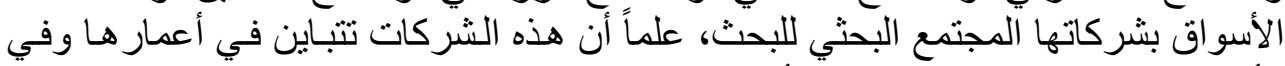
ر أسمالها في السوق الواتها الحدة وفي الأسواق مجتمعة. 
اعتمد تحليل بيانات البحث على عدد من النمـاذج المستخدمة في قياس إدارة العوائد

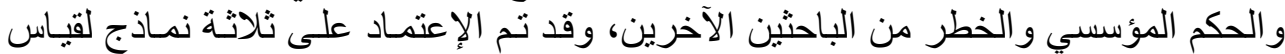

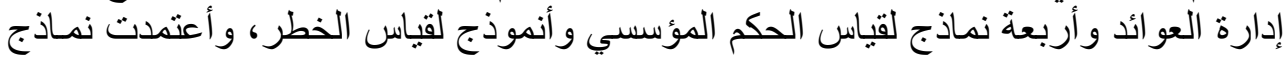

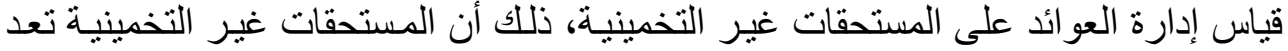

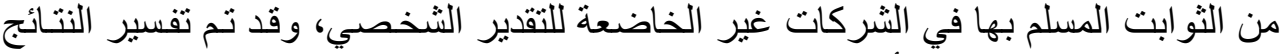
المتولدة من تطبيق النماذج أعلاه لتقييم الجانب التطبيقي في البحث و هذه النماذج هي:

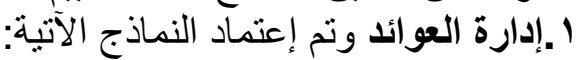

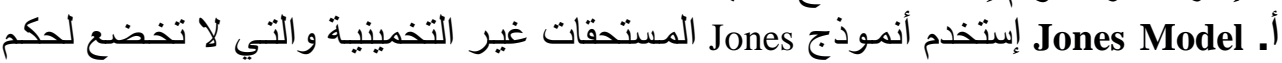
وسيطرة مدر اء الثركة ليتم التلاعب بها وذللك باستخدام الصيغة الآتية:

Non Discretionary Accruals $=a_{1}\left(1 /\right.$ Assets $\left._{i t-1}\right)+a_{2}\left(\Delta\right.$ Revenue $_{i t} /$ Assets $\left._{\text {it- }-1}\right)+a_{3}($ Gross property plant and Equipment $t_{\mathrm{it}}$ )

$\mathrm{NDA}_{\mathrm{it}}=\mathrm{a}_{1}\left(1 / \mathrm{A}_{\mathrm{it}-1}\right)+\mathrm{a}_{2}\left(\Delta \mathrm{REV}_{\mathrm{it}} / \mathrm{A}_{\mathrm{it}-1}\right)+\mathrm{a}_{3} \mathrm{PPE}_{\mathrm{it}}$ ويعتمد الأنموذج على المتغيرات التالية:

$\mathrm{NDA}_{\mathrm{it}}=$ Non Discretionary Accurals $\mathrm{it}_{\mathrm{it}}=$

$$
\text { المستحقات غير التخمينية للمنظمة i في سنة t. }
$$

$\mathrm{A}_{\mathrm{it}-1}=$ Assets $_{\mathrm{it}-1}=$

$\Delta \mathrm{REV}_{\mathrm{it}}=\Delta$ Revenue $_{\mathrm{it}}=$

$$
\text { إجمالي الموجودات للمنظمة i في السنة السابقة }
$$

$$
\text { التغير ات السنوية في إير ادات المنظمة i من سنة t-1 إلى سنة t.t. }
$$

$\mathrm{PPE}_{\mathrm{it}}=$ Gross Property Plant and equipment $\mathrm{it}_{\mathrm{it}}=$

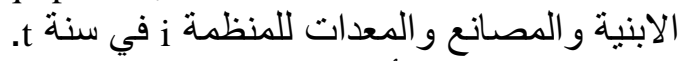

a a

بodified Jones Model

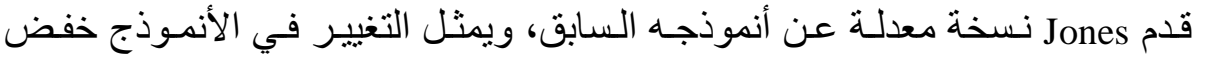

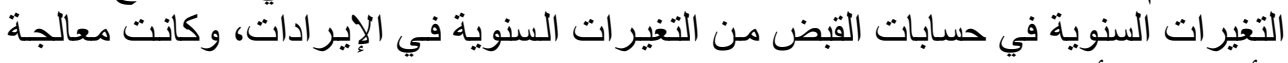

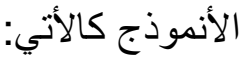

Non Discretionary Accruals $\mathrm{it}_{\mathrm{it}}=\mathrm{a}_{1}\left(1 /\right.$ Assets $\left._{\mathrm{it}-1}\right)+\mathrm{a}_{2}\left[\left(\Delta\right.\right.$ Revenue $_{\mathrm{it}}-\Delta$ Accounts

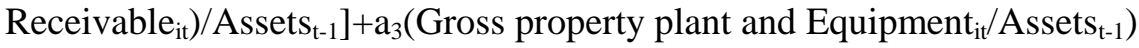
$\mathrm{NDA}_{\mathrm{it}}=\mathrm{a}_{1}\left(1 / \mathrm{A}_{\mathrm{it}-1}\right)+\mathrm{a}_{2}\left[\left(\Delta \mathrm{REV}_{\mathrm{it}}-\Delta \mathrm{REC}_{\mathrm{it}}\right) / \mathrm{A}_{\mathrm{it}-1}\right]+\mathrm{a}_{3}\left(\mathrm{PPE}_{\mathrm{it}} / \mathrm{A}_{\mathrm{it}-1}\right)$

$\mathrm{NDA}_{\mathrm{it}}=$ Non Discretionary Accurals $\mathrm{it}_{\mathrm{it}}=$

$$
\text { إذ يعتمد الأنموذج المعدل على المتغيرات الآتية: }
$$

$$
\begin{aligned}
& \text { المستحقات غير التخمينية للمنظمة i في سنة t. } \\
& \mathrm{A}_{\mathrm{it}-1}=\text { Assets }_{\mathrm{it}-1}=
\end{aligned}
$$$$
\text { اجمالي الموجودات للمنظمة i في السنة السابقة }
$$

$$
\begin{aligned}
& \Delta \mathrm{REV}_{\mathrm{it}}=\Delta \text { Revenue }_{\mathrm{it}}= \\
& \text { التغير ات السنوية في إير ادات المنظمة i من سنة t-1 إلى سنة t. }
\end{aligned}
$$

$\Delta$ REC $_{\mathrm{it}}=\Delta$ Accounts Recivable $_{\mathrm{it}}=$

$$
\text { التغير ات السنوية في حسابات القبض للمنظمة i من سنة t-1 إلى سنة t }
$$

$\mathrm{PPE}_{\mathrm{it}}=$ Gross Property Plant and equipment $\mathrm{i}_{\mathrm{it}}=$

$$
\text { الابنية و المصانع و المعدات للمنظمة i في سنة it. }
$$




\section{الجميل والحسيني] [VIV]}

a a

The Industrial Model $\rightarrow$

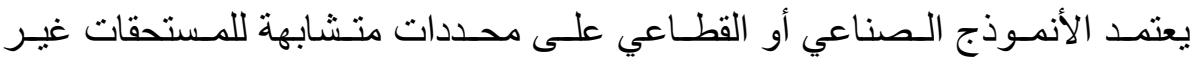

التخمينية في الثركات التي تنتمي لذات الصناعة، وقد تم إستخدام الصيغة الآتية:

Non Discretionary Accruals $\mathrm{s}_{\mathrm{it}}=\mathrm{a}+\mathrm{b}$ median(Total Accruals $_{\mathrm{it}} /$ Assets $_{\mathrm{it}-1}$ )

$\mathrm{NDA}_{\mathrm{it}}=\mathrm{a}+\mathrm{b}$ median $\left(\mathrm{TA}_{\mathrm{it}} / \mathrm{A}_{\mathrm{it}-1}\right)$

$\mathrm{NDA}_{\mathrm{it}}=$ Non Discretionary Accurals $\mathrm{it}_{\mathrm{it}}=$

$$
\text { وتتكون متغيرات الأنموذج مما يأتي: }
$$

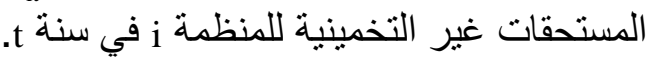

Median=الوسيط من خلال ترتيب القيم تصاعديا ثم اختيار القيمة الوسطية فئة

TA $_{\mathrm{it}}=$ Total Accruals it $=$

$\mathrm{A}_{\mathrm{it}-1}=$ Assets $_{\mathrm{it}-1}=$

$$
\text { اجمالي المستحقات للمنظمة i في سنة t. - اجن }
$$

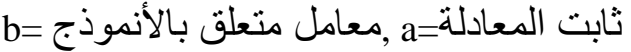

$$
\text { اجمالي الموجودات للمنظمة i في السنة السابقة }
$$

وقد تم احتساب المستحقات غير التخمينيـة للنمـاذج السابقة بحسب الأنمـوذج الذباني

Non Discretionary Accruals $\mathrm{Ait}_{\mathrm{it}}=$ Total Accruals $_{\mathrm{it}} /$ Assets $_{\mathrm{it}-1}$

طرحه De Angelo وكما في المعادلة الآتية: $\mathrm{NDA}_{\mathrm{it}}=\mathrm{TA}_{\mathrm{it}} / \mathrm{A}_{\mathrm{it}-1}$

$\mathrm{NDA}_{\mathrm{it}}=$ Non Discretionary Accurals $_{\mathrm{it}}=$

$$
\text { إذ يعتمد الأنموذج على المتغير ات الآتية: }
$$

$\mathrm{TA}_{\mathrm{it}}=$ Total Accruals $\mathrm{it}=$

$$
\text { المستحقات غير التخمينية للمنظمة i في سنة t. }
$$

$$
\text { اجمالي المستحقات للمنظمة i في سنة t. }
$$

$\mathrm{A}_{\mathrm{it}-1}=$ Assets $_{\mathrm{it}-1}=$

$$
\text { اجمالي الموجودات للمنظمة i في السنة السابقة }
$$

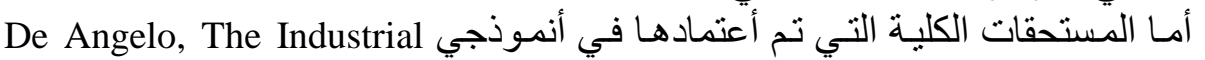

Total Accruals $s_{i t}=\Delta$ Accounts Receivable $_{i t}+\Delta$ Inventories $_{\text {it }}+\Delta$ Accounts Payable $_{i t}+$ Depreciation Expense $\mathrm{it}_{\mathrm{it}}$

$\mathrm{TA}_{\mathrm{it}}=\Delta \mathrm{REC}_{\mathrm{it}}+\Delta \mathrm{INV}_{\mathrm{it}}+\Delta \mathrm{AP}_{\mathrm{it}}+\mathrm{DE}_{\mathrm{it}}$

$\mathrm{TA}_{\mathrm{it}}=$ Total Accruals $\mathrm{sit}_{\mathrm{it}}=$

$$
\text { حيث: }
$$

$\Delta \mathrm{REC}_{\mathrm{it}}=\Delta$ Accounts $^{\mathrm{Recivable}} \mathrm{it}=$

$$
\text { اجمالي المستحقات للمنظمة i في سنة t. }
$$

$$
\text { التغير ات السنوية في حسابات القبض للمنظمة i من سنة t-1 إلى سنة t }
$$

$\Delta \operatorname{Inv}_{\mathrm{it}}=\Delta$ Invetoriles $_{\mathrm{it}}=$

$$
\text { التغير ات السنوية في المخزون للمنظمة i من سنة t-1 إلى سنة t. }
$$

$\Delta \mathrm{AP}_{\mathrm{it}}=\Delta$ Accounts Payable $_{\mathrm{it}}=$

$$
\text { التغير ات السنوية في حسابات الدفع للمنظمة i من سنة t-1 إلى سنة th. }
$$

$\mathrm{DE}_{\mathrm{it}}=$ Depreciation Expense $_{\mathrm{it}}=$ 


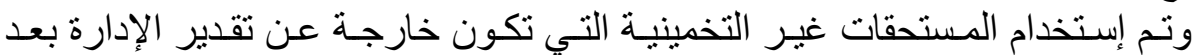

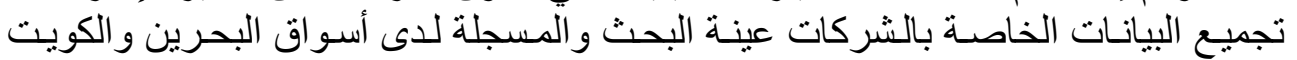

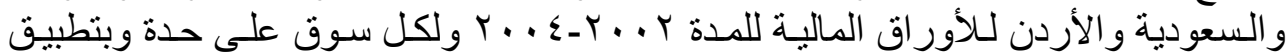
أسلوب Pooled Regression تم تنفيذ الصيغة التالية:

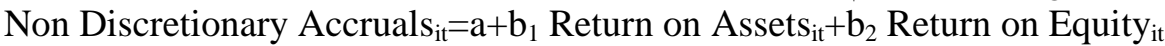
$\mathrm{NDA}_{\mathrm{it}}=\mathrm{a}+\mathrm{b}_{1} \mathrm{ROA}_{\mathrm{it}}+\mathrm{b}_{2} \mathrm{ROE}_{\mathrm{it}}$

ROA $_{\mathrm{it}}=$ Return on Assest $_{\mathrm{it}}=$

$$
\text { ويعتمد الأنموذج على المتغيرات الآتية: }
$$

ROE $_{\mathrm{it}}=$ Return on Equity $_{\mathrm{it}}=$ نسبة العائد على الموجودات للمنظمة i في سنة t.

$b_{1}, b_{2}=$ معلمات الأنموذ نسبة العائد على حق الملكية للمنظمة i في سنة t. a=ثابت المعادلة a

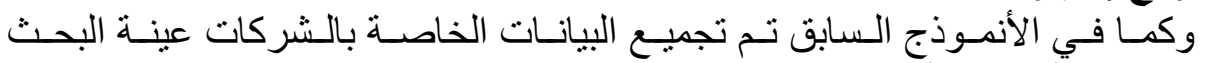
ب. أنموذج إختبار هيكل المالكين

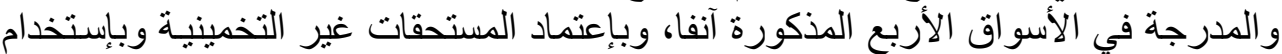

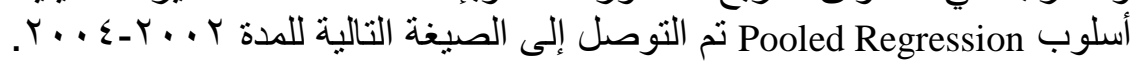
Non Discretionary Accruals $\mathrm{it}_{\mathrm{it}}=\mathrm{a}+\mathrm{b}_{1}$ Equity $_{\mathrm{it}}+\mathrm{b}_{2}$ Number of Shares $\mathrm{s}_{\mathrm{it}}+\mathrm{b}_{3}$ Earning per share $_{\text {it }}$

$\mathrm{NDA}_{\mathrm{it}}=\mathrm{a}+\mathrm{b}_{1} \mathrm{E}_{\mathrm{it}}+\mathrm{b}_{2} \mathrm{NS}_{\mathrm{it}}+\mathrm{b}_{3} \mathrm{EPS}_{\mathrm{it}}$

$\mathrm{E}_{\mathrm{it}}=$ Equity $_{\mathrm{it}}=$

$$
\text { إذ يعتمد الأنموذج على المتغيرات الآتية: }
$$

$\mathrm{NS}_{\mathrm{it}}=$ Number of Shares $_{\mathrm{it}}=$ حق الملكية للمنظمة i في سنة عدد الأسهم للمنظمة i في سنة

EPS $_{\mathrm{it}}=$ Earining per share $_{\mathrm{it}}=$

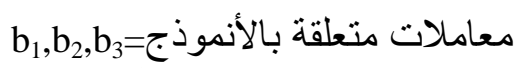

$$
\text { عائد السهم الو احد للمنظمة i في السنة t. }
$$

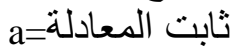

جـ. أنموذج إختبار عوائد ومكافآت الإدارة

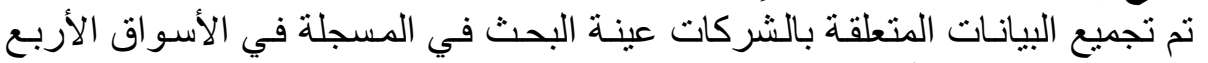

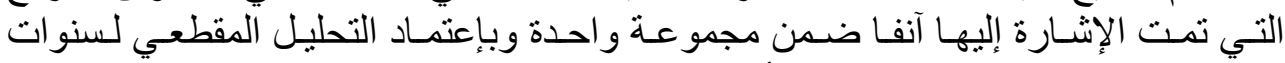

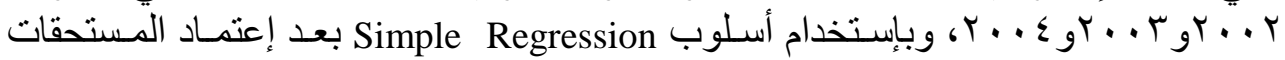
Non Desicretionary Accruals $s_{i t}=a+b_{1}$ Premium of Board $_{i t}$ غير التخمينية تم تتفيذ الصيغة الآنية: 


\section{الجميل والحسيني[Vr}

$\mathrm{NDA}_{\mathrm{it}}=\mathrm{a}+\mathrm{b} \mathrm{Pb}_{\mathrm{it}}$

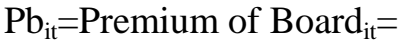

$$
\text { وتتكون متغير ات الأنموذج مما يأتي: }
$$

مكافأة اعضاء مجلس الإدارة للمنظمة i في سنة t.

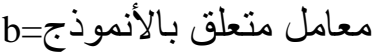

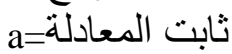

$$
\text { د. أنموذج إختبار إفصاح الشركات }
$$

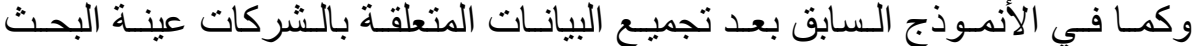

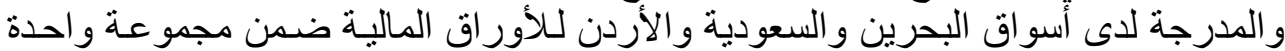

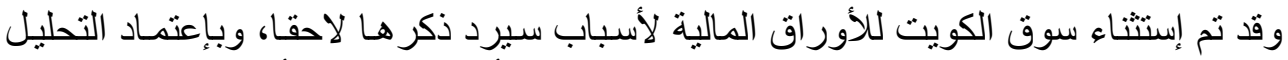

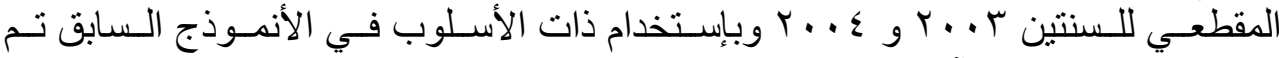

Price of share $_{\mathrm{it}}=\mathrm{a}+\mathrm{b}$ Equity on Number of shares

$\mathrm{PS}_{\mathrm{it}}=\mathrm{a}+\mathrm{bEON} \mathrm{N}_{\mathrm{it}}$

$\mathrm{PS}_{\mathrm{it}}=$ Price of share $_{\mathrm{it}}=$

$$
\text { ويعتمد الأنموذج على المتغيرات الآتية: }
$$

$$
\text { سعر سهم المنظمة i في سنة t. }
$$

$\mathrm{EON}_{\mathrm{it}}=$ Equity on Number of shares $_{\mathrm{it}}=$

$$
\text { نسبة حق الملكية إلى عدد الأسهم للمنظمة i في سنة t. }
$$

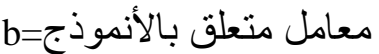

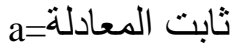

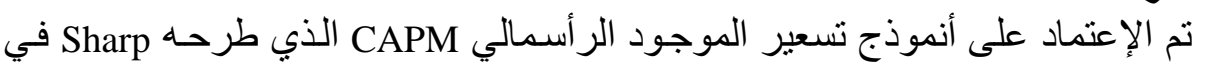

Expected Return $_{\mathrm{it}}=$ Risk-Free Return+Beta[(Market Return)-(Risk-Free Return)]

$\mathrm{ER}_{\mathrm{it}}=\mathrm{RF}+\mathrm{B}[\mathrm{RM}-\mathrm{RF}]$

ERit=Expected Return $_{\mathrm{it}}=$

$$
\text { معدل العائد المتوقع على الموجود (السهم) للمنظمة i في سنة t. }
$$

$\mathrm{RF}=$ Risk-Free Return $=$

$$
\text { معدل العائد الخالي من المخاطر. }
$$

RM=Market Return=

$$
\text { معدل عائد السوق او معدل العائد على محفظة موجودات السوق. }
$$

B=Beta= معامل بيتاو هو مؤشر للمخاطر غير القابلة للتنويع معات

ع ـأنموذج إختبار إدارة العوائد والحكم المؤسسي والخطر

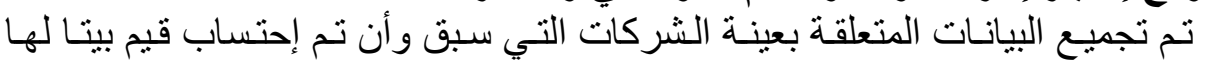

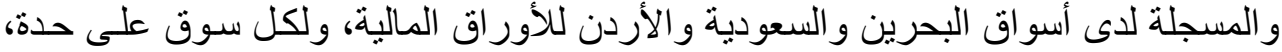

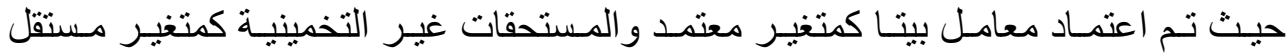

وبإستخدام أسلوب الإنحدار الخطي البسيط تم تنفيذ الصيغة الآتية:

Beta $_{\mathrm{it}}=\mathrm{a}+\mathrm{bNon}$ Discretionary Accruals $\mathrm{it}_{\mathrm{it}}$ 
$\mathrm{B}_{\mathrm{it}}=\mathrm{a}+\mathrm{bNDA}_{\mathrm{it}}$

$\mathrm{B}=$ Beta Confficient $\mathrm{it}_{\mathrm{i}}=$

b= معاملات متعلقة بالأنموذج

$$
\text { حيث: }
$$

معامل بيتا للمنظمة i في سنة t.

a=ثابت المعادلة

ويعد من الأدوات المهمة التي إعتمدها البحث لتحليل العلاقة بين المتغير ات المعتمدة

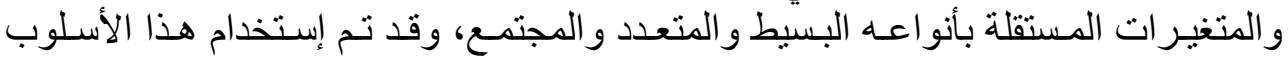

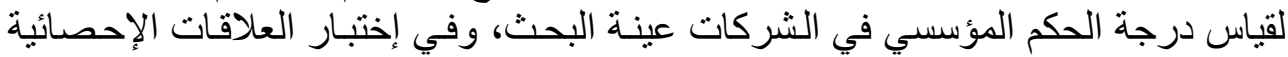
بين إدارة العو ائد والحكم المؤسسي والحي الخطر.

\section{مناقشة نتائج تحليل العلاقة بين إدارة العوائد والحكم المؤسسي والخطر}

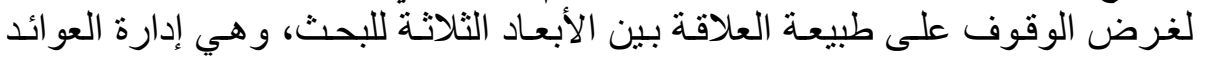

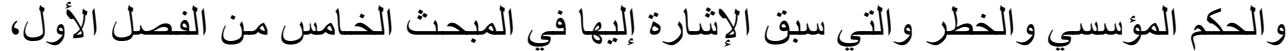

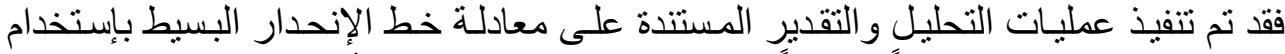

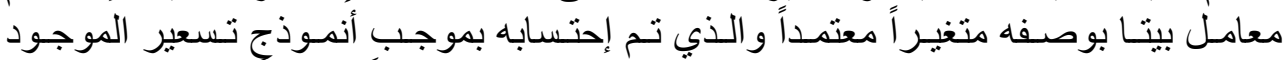

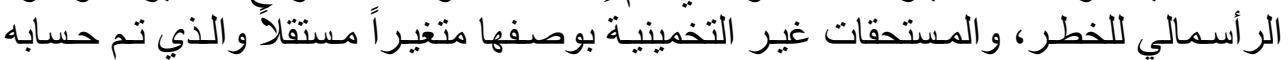

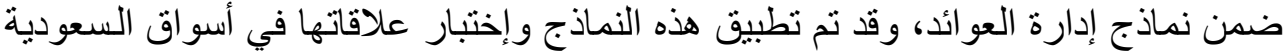

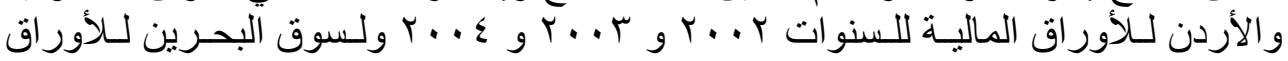

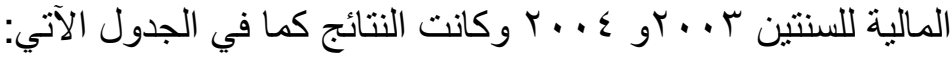

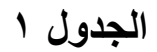

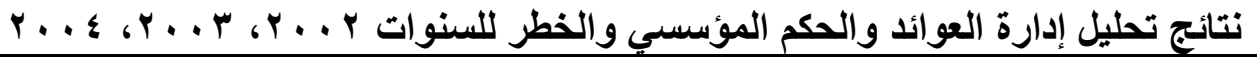

\begin{tabular}{|c|c|c|c|c|c|}
\hline Market & Year & $\mathbf{X}_{0}$ & $X_{1}$ & $\mathbf{R}^{2}$ & $\mathbf{F}$ \\
\hline \multirow{4}{*}{ Bahrain } & \multirow{2}{*}{2003} & 0.218 & 2.87 & \multirow{2}{*}{0.52} & \multirow{2}{*}{$6.42 * *$} \\
\hline & & $(0.53)^{\mathrm{N} . \mathrm{S}}$ & $(2.53)^{* *}$ & & \\
\hline & \multirow{2}{*}{2004} & -0.121 & 0.203 & \multirow{2}{*}{0.10} & \multirow{2}{*}{$0.69^{\text {N.S }}$} \\
\hline & & $(-1.78)^{\mathrm{N} . \mathrm{S}}$ & $(0.83)^{\text {N.S }}$ & & \\
\hline \multirow{6}{*}{ Saudi Arabia } & \multirow{2}{*}{2002} & 0.0607 & -0.294 & \multirow{2}{*}{0.28} & \multirow{2}{*}{$1.58^{\text {N.S }}$} \\
\hline & & $(3.25)^{* *}$ & $(-1.26)^{\mathrm{N} . \mathrm{S}}$ & & \\
\hline & \multirow{2}{*}{2003} & 0.216 & 4.483 & \multirow{2}{*}{0.48} & \multirow{2}{*}{$3.74^{\mathrm{N} . \mathrm{S}}$} \\
\hline & & $(1.02)^{\mathrm{N} . \mathrm{S}}$ & $(1.93)^{\mathrm{N.S}}$ & & \\
\hline & \multirow{2}{*}{2004} & 0.988 & -0.697 & \multirow{2}{*}{0.18} & \multirow{2}{*}{$0.87^{\text {N.S }}$} \\
\hline & & $(4.66)^{\mathrm{N} . S}$ & $(-0.93)^{N . S}$ & & \\
\hline \multirow[t]{4}{*}{ Jordan } & \multirow{2}{*}{2002} & 0.785 & -0.34 & \multirow{2}{*}{0.004} & \multirow{2}{*}{$0.07^{\text {N.S }}$} \\
\hline & & $(5.16)^{* * *}$ & $(-0.26)^{\mathrm{N} . \mathrm{S}}$ & & \\
\hline & \multirow{2}{*}{2003} & 0.194 & 1.02 & \multirow{2}{*}{0.04} & \multirow{2}{*}{$0.71^{\text {N.S }}$} \\
\hline & & $(0.56)^{\mathrm{N} . \mathrm{S}}$ & $(0.84)^{\text {N.S }}$ & & \\
\hline
\end{tabular}


الجميل والحسيني]

\begin{tabular}{|c|c|c|c|c|c|}
\hline Market & Year & $\mathbf{X}_{\mathbf{0}}$ & $\mathbf{X}_{\mathbf{1}}$ & $\mathbf{R}^{2}$ & $\mathbf{F}$ \\
\hline \multirow{2}{*}{2004} & 0.614 & -0.441 & \multirow{2}{*}{0.07} & \multirow{2}{*}{$1.17^{\text {N.S }}$} \\
\cline { 3 - 4 } & & $(0.09)^{* * *}$ & $(-1.08)^{\text {N.S }}$ & \\
\hline
\end{tabular}

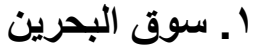

في ما يتعلق بالثركات المسجلة لدى سوق البحرين للأور اق المالية ولغرض التبر معرفة

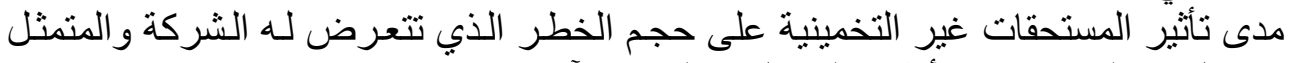
$\mathrm{Y}=1.218+2.87 \mathrm{X}$ بمعامل بيتا لسنة ؟ ... أظهر الجدول التيز النتائج الآتية:

$\mathrm{t}=(0.53)(2.53)$

$\mathrm{R}^{2}=52 \% \quad \mathrm{~F}=6.42$

يلاحظ من الجدول أعلاه و عند إستخدام إختبار (t) وجود علاقة ذات دلالة إحصائية

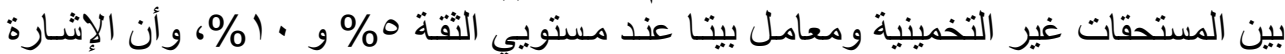

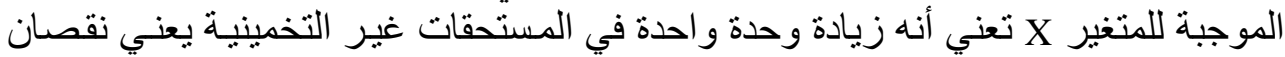

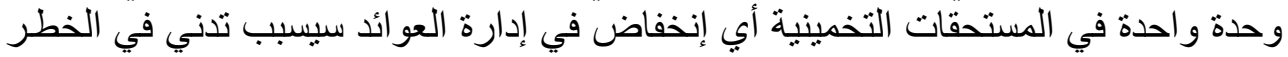

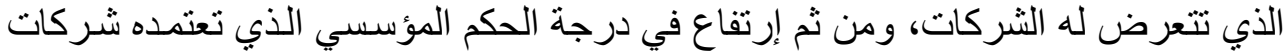

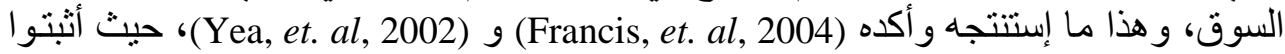

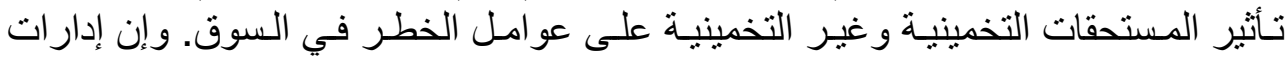

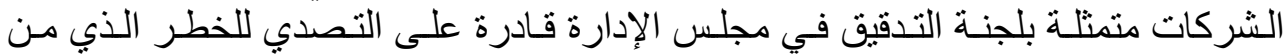

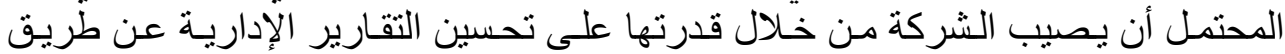

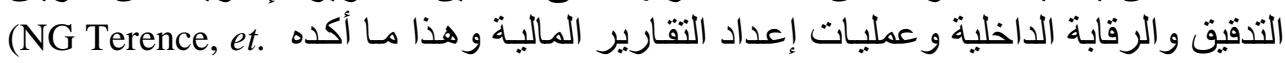
و أظهر الجدول ا أن قيمة F المحتسبة أكبر من قيمة نظيرتها الجدولية عند مستويي .al., 2001)

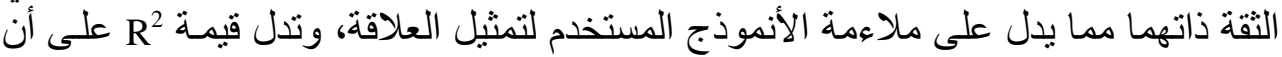

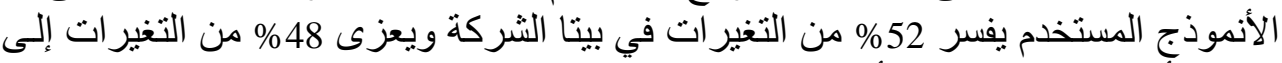

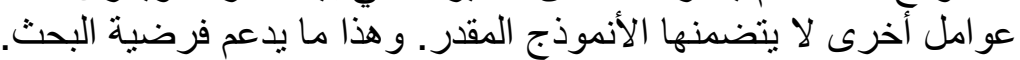
$\mathrm{Y}=-0.121+0.203 \mathrm{X}$

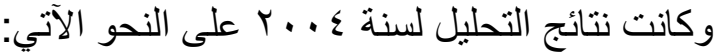

$\mathrm{t}=(-1.78)(0.83)$

$\mathrm{R}^{2}=10 \% \quad \mathrm{~F}=0.69$

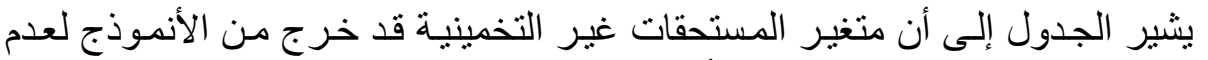

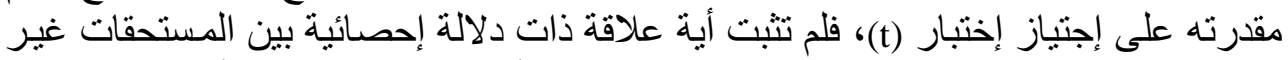

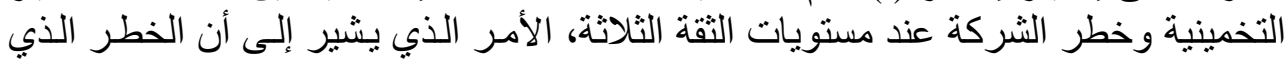

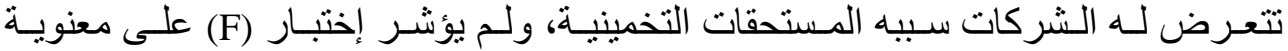

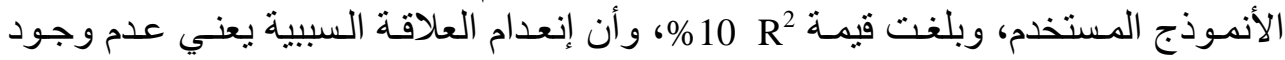

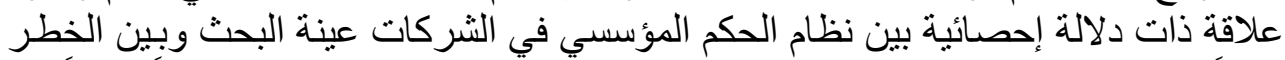

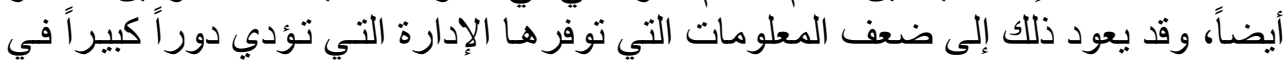

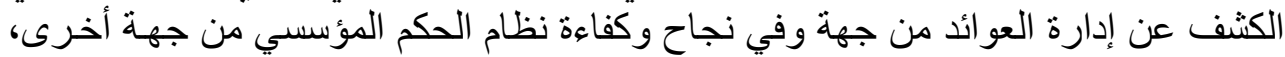


و هذا مـا أكده (Claro, et al, 2003) مما يثبت دور المستحقات التخمينية في قياس مخاطر الثركة في السوق من خلال بيتا.

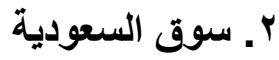

يتضح من نتائج تطبيق أنموذج الإنحدار الخطي البسيط على الثركات المسجلة لدى الدي

$\mathrm{Y}=0.0607-0.294 \mathrm{X}$

السوق السعودية لسنة ب ... T ما عرضته المعادلة الآتية :

$\mathrm{t}=(3.25) \quad(-1.26)$

$\mathrm{R}^{2}=28 \% \quad \mathrm{~F}=1.58$

$\mathrm{Y}=0.216+4.483 \mathrm{X}$

و وأثنارت نتائج التحليل لسنة ؟ ... إلى الآتي :

$\mathrm{t}=(1.02) \quad(1.93)$

$\mathrm{R}^{2}=48 \% \quad \mathrm{~F}=3.74$

$\mathrm{Y}=0.988-0.697 \mathrm{X}$

وكانت نتائج التحليل لسنة ؛ ... ع على النحو الآتي:

$\mathrm{t}=(4.66) \quad(-0.93)$

$\mathrm{R}^{2}=18 \% \quad \mathrm{~F}=0.87$

يتبين من الجدول إلى أن متغير المستحقات غير التخمينية ليس لـه تأثنير معنوي على إنى

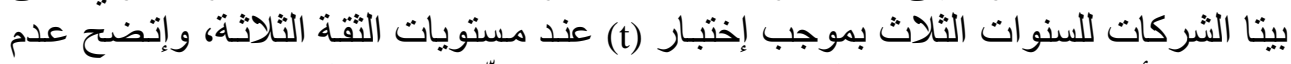

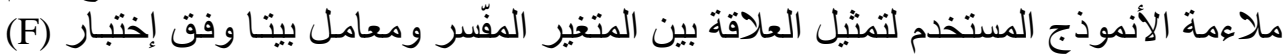

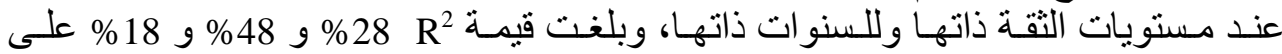

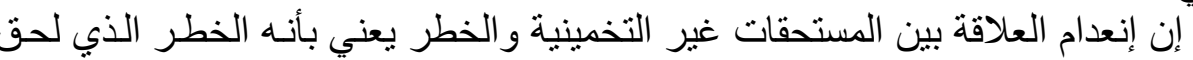

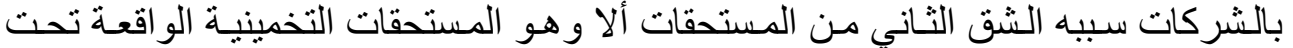

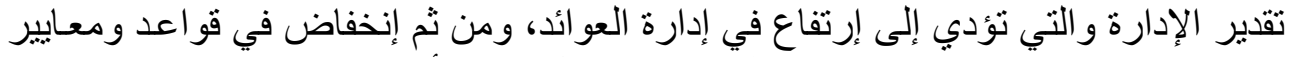

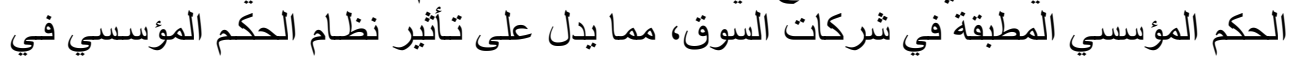

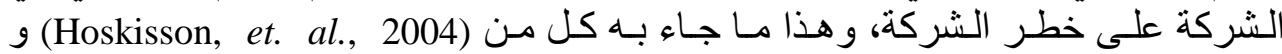

.(Salva,2003)

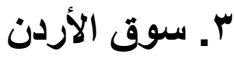

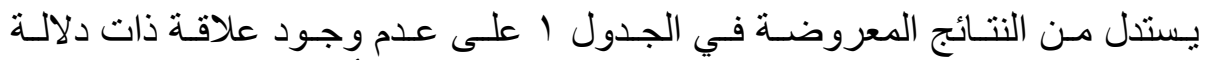

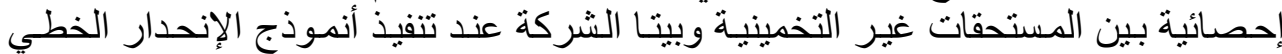

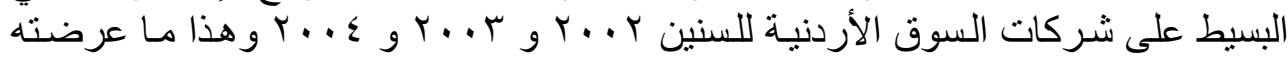

$\mathrm{Y}=0.785-0.34 \mathrm{X}$

$\mathrm{t}=(5.16) \quad(-0.26)$

$\mathrm{R}^{2}=0.4 \%$

$\mathrm{F}=0.07$

$\mathrm{Y}=0.194+1.02 \mathrm{X}$

$\mathrm{t}=(0.56) \quad(0.84)$

$\mathrm{R}^{2}=4 \%$

$\mathrm{F}=0.71$

$\mathrm{Y}=0.614-0.441 \mathrm{X}$

$\mathrm{t}=(0.09) \quad(-1.08)$ 


\section{الجميل والحسيني]}

$\mathrm{R}^{2}=0.7 \% \quad \mathrm{~F}=1.17$

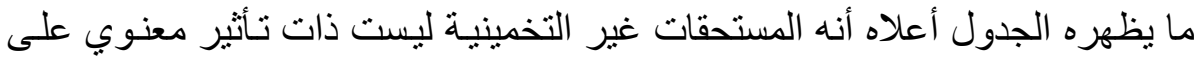

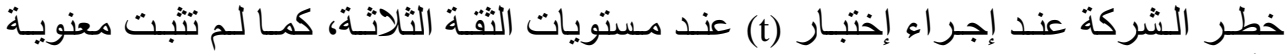

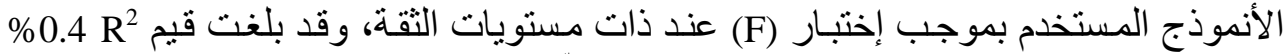

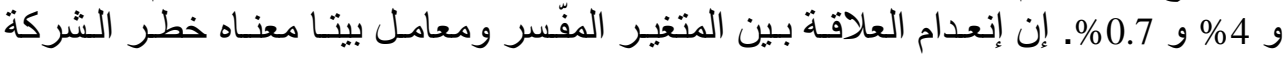

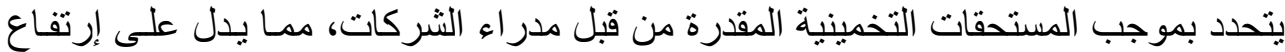

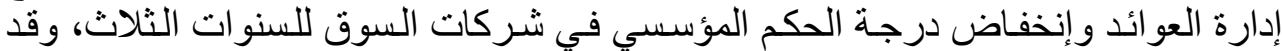

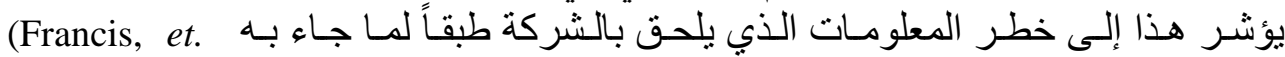

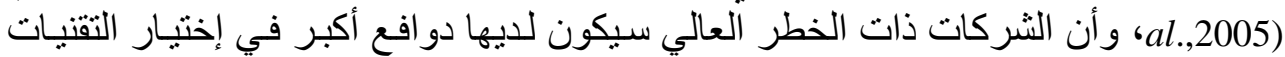
المحاسبية التي تستطيع من خلالها أن تتجنب الخسائر التي قد تصبيها (Yea, et. al, 2002). بناء على ما تقام تم دحض فرضية التية البحث.

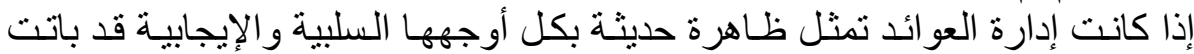

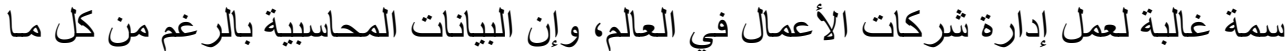

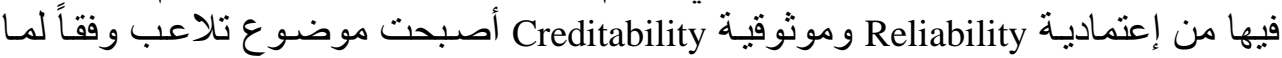
تقتضيه مصلحة الإدارة التنفيذية للشركة، فبرز الحكم المؤسسي Governance كنظام وهيك وهيكل مؤسسي و عمليات و أدوات ليضع الإدارة التنفيذية موضع المساءلة ولة والثفافية والإفصاح.

الخاتمة

توصل البحث إلى أنه يمكن الوصول إلى صيغة لنمذجة العلاقة بين الحكم المؤسسي

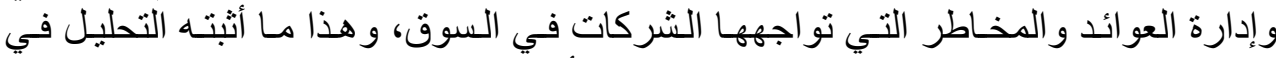

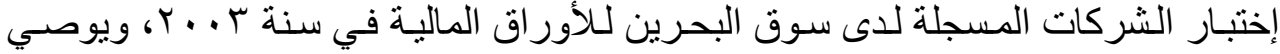

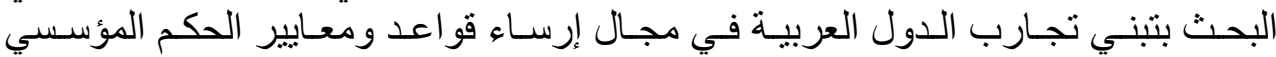

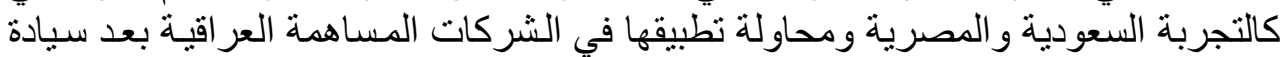

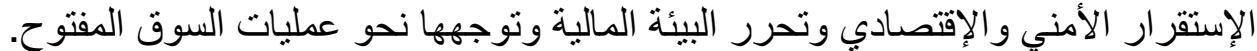

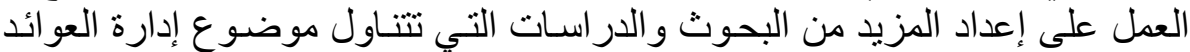

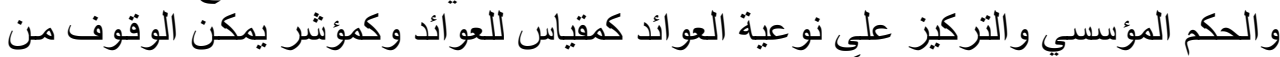

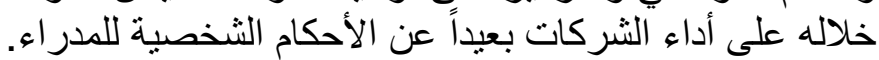

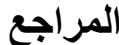

1. Babalyan Levon, 2004, "Earning Management by Firms Applying International Financial Reporting Standards Implications for Valuation", Ph.D. Thesis, Switzerland.

2. Baralexis Spyros, 2004, "Creating Accounting in Small Advancing Countries", the Greek Case Managerial Auditing Journal, Vol.19, No.3.

3. Beneish Messod D., 2001, "Earning Management: Aperspective", Managerial Finance, Vol.27, No. 12.

4. Burgstahler D. and Dichev I., 1997, " Earning Management to Avoid Earning Decrease and Losses", Journal of Accounting and Economics, 24.

5. Chin Stephen W.K., Hung Evap. W., 2004, "Good Governance or Muddling Through? Layoffs and Employment Retormin Socialist China", Communist and Post Communist Studies $\mathrm{xx}, \mathrm{xxx}-\mathrm{xxx}$.

6. Chirinko Robert S. and Schaller Huntely,2004, "Arevealed Perforence approach of Understanding Corporate Governance Problems : evidence from Canada", Journal of Financial Economics, Oct. 
7. Claro Danny Pimentel, Hagelaar Geoffrey and Omta Onno, 2003, "The determinates of relational governance and performance : How to manage business relationships?", Industrial Marketing Management, 32.

8. Davidson R., Goodwin - Stewart J. and Kent P., 2005, "The Internal Structure and Earnings Management", Accounting and Finance, Vol.45.

9. De Angelo, 1986, "Accounting Numbers as Market Valuation Substitutes : A Study Management Buyout of Public Shareholders", The Accounting Review, 61.

10. Dechow and Dichev, 2002, "The Quality of Accruals and Earnings, the Role of Accruals Estimation Errors", The Accounting Review, 77(4).

11. Dechow Partricia M., Sloan Richard G. And Sweeney Amy P., 1995, "Detecting Earning Management", The Accounting Review, Vol.70, No.2, April.

12. Economist, 2001, "Economic Focus, Taking the Measure" The Economist, November, 24.

13. Francis Jennifer, Lafond Ryan, Olsson Per and Schipper Katherine, 2004, "Costs of Equity and Earnings Attributes", The Accounting Review, 79(4).

14. Francis Jennifer, Lafond Ryan, Olsson Per and Schipper Katherine, 2005, "The Market Pricing of Accruals Quality", Journal of Accounting and Economics, 39.

15. Frensch Richard, 2004, "Public Governmance as a Source of Quality and Veraity Gains from Transition", Journal of Comparetine Economics, (exxx-xxx).

16. Hansel Dennis, Krahnen Jan Pieter and Wilde Christon, 2006, "Monitoring Credit Risk Transfer in Capital Markets : Statistical Implications", 3d ECB Conference on Statistics, May 4-5.

17. Healy, P.M, and J.M. Wahlen, 1999, "A review of the Earnings Management Literature and its implications for standard setting" Accounting Horizons, Vol.13, No.4.

18. Hoskisson Robert E., Yin Daphne and Kin Hicheon, 2004, "Corporate Governance Systems : Effects of Capital and Labbor Market Congruency on Corporate Innovation and Global Competitiveness", Journal of High Technology Management Research 15.

19. NG Terence bu - Peow, Green Wendy and Nett Roger Sim, 2001, "The effects of fraud risk and management representation on an Auditor's Hypothesis Generation", Abacus, Vol. 37, No.3.

20. Revsine, L., 1991, "The Selective Financial Misrepresentation Hypothesis", Accounting Horizons, December.

21. Salva, Corolina, 2003, "Foreign Listings, Corporate governance and equity valuations", journal of Economics and Business, 55.

22. Scripper, 1989, "Commentary on Earning Management, Accounting Horizons, Vol. 1.

23. Yea Gillian H.H., S. Patricia M., Waiho Kim and Chen Sheng - Syan, 2002, "Corporate Ownership Structure and the Informativeness of Earings", Journal of Business Finance \& Accounting, 29(7) \& (8), Spt./Oct.

24. Zhang Huai, 2002, "Detecting Earning Management - Evidence from Rounding - upin Reported EPS", Department of Accounting, College of Business Administration, University of Illinois at Chicago, USA.

25. Ziv, 1998, "A Discussion of Earning Management and the Reveluation Principle", Review of Accounting Studies, 3.

26. Gitman Lawrence J., 2000, "Principles of Managerail Finance", 9th ed ISBN. United States.Keasey Kevin, Thompson Steve and Wright Mike, 1997, "Corporate Governance Economic Management and Financial Issues", McGraw-Hill, USA.

27. Mulford Charles W., Comiskey Eugene E., 2002 "The Financial Numbers Game Detecting Creative Accounting Practices", John Wiley and Sons. INC., USA.

28. Pike Richard, Neale Bill, 1993, "Corporate Finance and Investement Decisions and Strategies", Prentice Hall International, U.K. Ltd, 2nd .ed., Prentice - Hall. 
الجميل والحسيني[V9] 